\title{
Competencia y competividad portuarias: una aplicación a las fachadas marítimas españolas*
}

\section{Port competition and competitiveness: An application to the Spanish seafronts*}

Núm. 4 (2015), pp. 59-85.

Villaverde Castro, José ${ }^{*_{1}}$

Maza Fernández, Adolfo * ${ }^{*}$

JEL Clasif: $\mathrm{O} 18$

DOI: $10.5944 /$ reppp.4.2015.13348

Este trabajo se ha beneficiado de los comentarios y sugerencias del profesor Pablo Coto, al que expresamos nuestro agradecimiento. Los posibles errores son responsabilidad exclusiva de los autores.

*1 José Villaverde, Departamento de Economía, Universidad de Cantabria; Kemmy Business School, University of Limerick, Limerick, Ireland. Email: villavej@unican.es

*2 Adolfo Maza, Departamento de Economía, Universidad de Cantabria. Email: mazaaj@unican.es 


\title{
Resumen
}

Los puertos españoles son uno de los principales elementos de la red de infraestructuras de transporte del país, sobre todo desde el punto de vista del transporte internacional. Por ello, en este trabajo se analiza, para el año 2007 y por fachadas marítimas, la competencia y competitividad portuarias. Con este fin se calculan una serie de indicadores ya conocidos, así como un nuevo indicador que, como novedad, incluye la distancia en el análisis. Los resultados muestran que las fachadas marítimas con mayor atractivo son la Surmediterránea, si hablamos en términos de valor, y la Norte, si lo hacemos en términos de peso. Por tipo de mercancía, la fachada Norte ocupa el primer lugar en graneles sólidos y mercancía general, mientras que la Surmediterránea ocupa tal posición en lo que se refiere a graneles líquidos, al tiempo que Levante sobresale como la fachada más atractiva en el tráfico de contenedores. Para finalizar, se incluyen algunas recomendaciones de política pública.

Palabras Clave: Puertos; fachadas marítimas; competencia; competitividad; atractivo; distancia

\begin{abstract}
Spanish ports are one of the main segments of the country infrastructure network, especially regarding to international trade. For this reason, this article analyses, for the year 2007 and for Spanish seafronts, port competition and competitiveness. To do it we compute some well-known indices, as well as a new indicator taking into account the role of distance. The results show that the most attractive seafronts are the SouthMediterranean, in terms of traffic value, and the North, in terms of traffic weight. By type of good, the North seafront is the most relevant in solid bulks and general cargo, while the South-Mediterranean seafront is the first one in liquid bulks and the Levante seafront highlights in container traffic. Finally, some recommendations about public policy are included.
\end{abstract}

Key Words: Ports; seafronts; competition; competitiveness; attractiveness; distance 


\section{Introducción}

Según datos recientes de la Secretaría de Estado de Comercio Exterior, alrededor del 30\% del valor de las exportaciones españolas, y cerca del 35\% de nuestras importaciones, se realizan a través del transporte marítimo. Estas cifras, en sí mismas muy significativas, aumentan considerablemente si los cálculos se realizan en términos de peso, donde el porcentaje de exportaciones e importaciones alcanza el $49 \%$ y $77 \%$, respectivamente. Dicho en otros términos, no se puede poner en duda, bajo ningún concepto, la importancia de los puertos españoles como uno de los principales elementos de la red de infraestructuras de transporte del país, sobre todo desde el punto de vista del transporte internacional y, en cuanto tales, de la competitividad de la economía nacional. En efecto, el transporte marítimo se elige como un eslabón clave de la cadena logística, más si cabe en años recientes, en los que los servicios marítimos han experimentado una considerable expansión impulsada por la creciente globalización de la economía mundial y la consiguiente desaparición de muchas políticas marítimas restrictivas.

En este contexto, el presente trabajo intenta efectuar una aproximación al muy interesante, pero un tanto elusivo, tema de la competencia y competitividad interportuaria. Con este objeto, y dado que el análisis puerto a puerto sería demasiado prolífico, se toman las fachadas marítimas españolas como objeto de estudio. Así, y siguiendo, entre otros, a García et al. (2007), en este trabajo se considera la existencia de ocho fachadas, cada una de las cuales está configurada por los puertos de interés general que se indican, a continuación de su nombre, entre paréntesis: Galicia (A Coruña, Ferrol-San Cibrao, Marín y Ría de Pontevedra, Vigo y Vilagarcía), Norte (Avilés, Bilbao, Gijón, Pasajes y Santander), Cataluña (Barcelona y Tarragona), Levante (Alicante, Cartagena, Castellón y Valencia), Surmediterránea (Almería, Bahía de Algeciras, Málaga, Motril, Ceuta y Melilla), Suratlántica (Bahía de Cádiz, Huelva y Sevilla), Baleares (Baleares) y Canarias (Las Palmas y Santa Cruz de Tenerife). Para cada una de estas fachadas se estudia, a partir de una Base de Datos Integrada de Puertos y Aduanas proporcionada por Puertos del Estado, el tráfico portuario correspondiente al año 2007 (en términos de peso y de valor), tanto a nivel agregado como desagregado en exportaciones e importaciones; en todos estos casos se examina, asimismo, no sólo el tráfico total sino su distribución en contenedores, graneles líquidos, graneles sólidos y mercancía general.

Con estas precisiones en mente, en la primera parte del trabajo, y como punto de partida, se efectúa una revisión de la literatura sobre la materia, mientras que en la segunda, su parte central, se calculan una serie de indicadores que, indirectamente, tratan de reflejar el grado de competencia y competitividad entre las mencionadas fachadas. Para ser más precisos, lo que estos indicadores ofrecen es una idea bastante acertada, creemos, del grado de atractivo de las distintas fachadas marítimas para todas y cada una de las provincias españolas. Como es habitual, en la última sección se presentan, con brevedad, las conclusiones más relevantes. 


\section{Competencia y competitividad portuaria: una revisión de la literatura}

\subsection{Competencia y competitividad: conceptos genéricos}

Como es de sobra conocido, cuando en economía se utiliza el término competencia se hace referencia a una situación en la que varios agentes actúan en un mismo mercado persiguiendo objetivos económicos más o menos similares. Si las características de los agentes fueran muy parecidas entre sí -lo que, en puridad técnica implicaría que ninguno de ellos tendría capacidad para imponer precios y cantidades- hablaríamos de una situación de competencia perfecta; si, por el contrario, hubiera diferencias significativas entre los agentes en lo que hace a su capacidad para influir sobre precios y cantidades, entonces estaríamos hablando de alguna variedad de competencia imperfecta.

El término competitividad, por su parte, surge a raíz de la existencia de competencia. Si no hubiera competencia entre agentes, estos no tendrían ninguna necesidad de ser competitivos ya que operarían en lo que, para entendernos, podemos denominar como mercados cautivos. En realidad, sin embargo, casi siempre existe algún grado de competencia (aunque ésta prácticamente nunca sea perfecta), lo que implica que, para hacerse con una cierta posición (cuota) en el mercado es necesario que los agentes sean competitivos, tanto más cuanto que el nuevo contexto de globalización económica ha traído consigo crecientes niveles de competencia.

En este sentido, hay que subrayar que el análisis de la competitividad no sólo despierta el interés académico sino que, de forma creciente, se está convirtiendo en motivo de debate y de toma de posición desde perspectivas políticas muy variadas. $\mathrm{Al}$ respecto hay que subrayar que la Comisión Europea (European Commission, 2004) considera que la mejora del grado de competitividad de las regiones más atrasadas constituye un elemento vital para el logro de la cohesión social y territorial. No es de extrañar, por lo tanto, que sean legión los gobiernos interesados en conocer la posición competitiva de sus países, lo cual implica, siguiendo la conocida afirmación de Lord Kelvin de que «lo que no se puede medir no se puede mejorar», el interés por la elaboración de los indicadores correspondientes.

Dicho esto, la primera cuestión que debemos formularnos es la de qué se entiende por competitividad, un concepto que en los últimos tiempos ha pasado de la esfera empresarial (microeconómica) a la nacional (macroconómica). De acuerdo con Budd y Hirmis (2004), el vocablo «competitividad», al igual que el de «globalización», es un término que arroja mucho calor pero poca luz; se utiliza con demasiada frecuencia en el ámbito económico, pero, en general, es poco esclarecedor. Pese a lo que de cierto pueda tener esta opinión, hay que convenir (véanse, por ejemplo, Kitson et al., 2004, y Turok, 2004) que competitividad es un término que está relativamente bien definido a escala empresarial; cosa distinta, sin embargo, es su definición desde una perspectiva macroeconómica. A nivel agregado, quizás la definición más interesante sea la ofrecida por la Comisión Europea, que entiende la competitividad como «la capacidad para producir bienes $y$ servicios que superen la prueba de los mercados internacionales y que mantengan al mismo tiempo unos niveles elevados y duraderos de renta o, en términos más generales, 
la capacidad de las empresas, los países y las regiones supranacionales para generar, estando expuestas a la competencia internacional, unos niveles de renta y de empleo relativamente altos» (Comisión Europea, 1999, p. 75).

En esta definición subyace el nexo evidente que existe entre la competitividad microeconómica y la competitividad macroeconómica, siendo la primera uno de los puntos de partida para la existencia de la segunda. Así, y pasando del nivel macroeconómico (que habla básicamente de competencia y competitividad entre países o regiones) al microeconómico, la competitividad empresarial se puede definir como la capacidad de las empresas para ganar cuota de mercado consiguiendo la mayor satisfacción posible de los consumidores al menor precio.

Ahondando en lo anterior, la competitividad a nivel microeconómico depende, en esencia, de dos factores: el precio y la calidad del producto o servicio. Existen, además, otros factores que también afectan a la competitividad, entre los que la imagen corporativa del productor ha ido adquiriendo un rol preponderante. Adicionalmente, cabe indicar que, como señala Winkelmans (2002), las empresas no sólo compiten con bienes o factores productivos tangibles, sino que también lo hacen en servicios intangibles que proporcionan alto valor añadido. Por ello, cada vez tienen más importancia las ventajas competitivas apoyadas en los activos intangibles de las empresas.

\subsection{Competencia y competitividad portuarias}

Con referencia al mundo del transporte, y en particular al del transporte marítimo, conviene empezar precisando que la globalización económica ha impuesto nuevas reglas de juego y una redistribución mundial de los factores de producción tradicionales. La existencia de corredores de transporte ha abaratado sobremanera los costes de desplazamiento de mercancías lo que, unido al descenso de los costes de las comunicaciones, ha favorecido los intercambios marítimos internacionales. Estos, a su vez, se han visto incrementados porque en los últimos años hemos asistido a una creciente liberalización del comercio internacional y a una importante estandarización de los productos y del tráfico de contenedores.

En el ámbito marítimo es necesario distinguir entre competencia y colaboración portuaria, ya que si bien es cierto que el proceso de globalización ha favorecido la competencia en determinados aspectos, también lo es que ha favorecido la cooperación o colaboración en otros.

La coopetition es un neologismo que hace referencia a la «cooperative competition», esto es, a la «competencia cooperativa» (Cerbán, 2009). La competencia cooperativa, o «colaborar para competir», es un término ya empleado por Von Neumann (1944) en su Theory of Games and Economic Behavior. El término coopetition ha sido utilizado recientemente por Noorda (1993) para definir una estrategia de las empresas que constituye una mezcla entre competencia y colaboración (co-opetition, es el resultado de la combinación de los términos «competition» $\mathrm{y}$ «co-operation»), de tal modo que las empresas con un mismo objetivo o mercado apuesten por la estrategia de ganar siempre, compitiendo en algunos aspectos y colaborando en otros. La estrategia habitual en estas empresas era la de competir hasta expulsar a la otra empresa del mercado; la estrategia alternativa, cada vez más implantada, es la de que varias empresas actúen estratégicamente como una sola empresa. 
Hablamos de competencia portuaria, pero aún no hemos definido qué se entiende por tal. Existen, el respecto, diferentes definiciones, siendo la más sencilla y natural la que la asimila a la lucha de los puertos entre sí para captar más mercancías que el resto y/o para captar tráficos con más valor por unidad de mercancía, pues así el impacto económico de los mismos será mayor. Siguiendo a Van der Voorde y Winkelmans (2002), Cerbán (2009) distingue cuatro tipos de competencia portuaria:

1. Competencia entre empresas de un puerto.

2. Competencia entre puertos.

3. Competencia entre grupos de puertos (puertos con características geográficas comunes compiten entre ellos).

4. Competencia entre rangos portuarios (puertos localizados a lo largo de la misma costa o con un hinterland prácticamente idéntico compiten con los de otro rango por unos tráficos determinados).

En cuanto a los factores que explican la competitividad portuaria, Yanbing y Zhongzhen (2005) especifican los mismos para el caso concreto del tráfico de contenedores. Estos factores, que son muy similares a los apuntados por Fleming y Baird (1999) para todo tipo de tráficos, se resumen, en esencia, en los siguientes:

1. Tradición portuaria.

2. Rango portuario (puertos que comparten una misma costa y un mismo hinterland).

3. Accesibilidad portuaria.

4. Apoyo público.

5. Productividad portuaria.

6. Preferencias de los agentes hacia los puertos.

7. Localización geográfica.

\subsection{Las ventajas competitivas de los puertos: métodos de estimación}

Para determinar y definir las ventajas competitivas de los puertos existen diferentes metodologías, entre las que cabe destacar las cuatro siguientes:

1. Métodos basados en los estudios de productividad y eficiencia de los puertos en competencia.

2. Métodos basados en los estudios de impacto económico portuario.

3. Métodos basados en los estudios de las matrices de competitividad de los puertos en competencia.

4. Métodos basados en los estudios de las desviaciones de los tráficos, hinterland y foreland de los puertos en competencia.

A continuación hacemos una breve referencia a estas metodologías, si bien nuestro trabajo empírico va a basarse exclusivamente en la última. 


\subsubsection{Método basado en los estudios de productividad y eficiencia}

Los estudios de productividad y eficiencia proporcionan evidencia sobre las ventajas competitivas de un puerto respecto al resto. Entendiendo la productividad como la relación existente entre los outputs obtenidos y los inputs empleados para obtener dichos outputs, parece obvio que el estudio de las relaciones input-output y sus diferencias entre puertos constituye un buen enfoque para determinar las ventajas competitivas.

En el caso concreto de los puertos, considerados como productores de varios outputs (mercancía general, graneles sólidos, graneles líquidos, contenedores y pasajeros), la forma funcional que se suele seleccionar para la estimación de los costes es la función translogarítmica.

Desde la perspectiva de la eficiencia, el aspecto básico de la función de producción radica en que ésta proporciona la máxima cantidad de producto que es posible obtener para cada vector de cantidades aplicadas de los factores productivos. En este sentido, las medidas de eficiencia de los puertos se obtienen comparando los valores observados para cada unidad productiva con el óptimo definido por la frontera estimada.

La literatura sobre competencia en puertos utiliza el enfoque de productividad y eficiencia, medidas ambas a través de las fronteras de las funciones de producción y costes (Baños-Pino et al., 1999; Coto-Millán et al., 2000; y Becchetti et al., 2003). Las investigaciones sobre el tema introducen la función de producción y la función de costes, para valorar la magnitud de eficiencia técnica, asignativa y económica. Se parte de que cuanto más eficiente sea un puerto más competitivo será, dado que los outputs, los inputs y la tecnología son esencialmente los mismos en la mayoría de los puertos.

Centrándonos en la eficiencia económica, que aúna las eficiencias técnica y asignativa, su estudio se fundamenta en la estimación de una frontera de la función de costes que representa, en términos de datos observados, el coste mínimo de producir un nivel particular de output, dada la tecnología y los precios de los inputs de producción usados.

Pasando a la eficiencia técnica, ésta puede analizarse desde dos aproximaciones. La primera, llamada eficiencia técnica orientada a los inputs, adopta la perspectiva de que un puerto puede producir y observar el output usando la cantidad menor de recursos físicos posible (inputs). La segunda aproximación, eficiencia técnica orientada a los outputs, se refiere a la posibilidad de incrementar el output ó outputs de los puertos sin cambios en la cantidad de inputs.

Un resumen de los principales trabajos sobre funciones de producción y costes que tratan de estudiar la productividad portuaria, estructura de costes, eficiencia técnica, económica y asignativa, y en definitiva la competitividad, con especial atención a los puertos españoles, se presenta en el Cuadro 1. González y Trujillo (2009) realizan una revisión de este tipo de literatura bastante completa, mientras que Kim y Sachis (1986) fueron los autores pioneros en estimar una función de producción en puertos con la finalidad de cuantificar el cambio técnico portuario. 


\section{Cuadro I}

Investigaciones empíricas sobre competitividad portuaria.

\begin{tabular}{|c|c|c|c|c|}
\hline Autores & $\begin{array}{l}\text { Especificación } \\
\text { Funcional }\end{array}$ & Datos & $\begin{array}{c}\text { Variables objeto de } \\
\text { atención }\end{array}$ & $\begin{array}{l}\text { Rendimientos } \\
\text { a Escala }\end{array}$ \\
\hline Chang (1978) & $\begin{array}{c}\text { Función de } \\
\text { producción Cobb- } \\
\text { Douglas }\end{array}$ & $\begin{array}{l}\text { Serie temporal } \\
(1953-1973) \\
\end{array}$ & $\begin{array}{c}\text { Productividades Medias y } \\
\text { Marginales }\end{array}$ & Constantes \\
\hline $\begin{array}{l}\text { Kim y Sachis } \\
\text { (1986) }\end{array}$ & $\begin{array}{l}\text { Función de costes } \\
\text { translog }\end{array}$ & $\begin{array}{l}\text { Serie temporal } \\
(1966-1983) \\
\end{array}$ & $\begin{array}{l}\text { Elasticidades de las demandas } \\
\text { de inputs }\end{array}$ & Crecientes \\
\hline $\begin{array}{l}\text { Rekres et al. } \\
\quad(1990)\end{array}$ & $\begin{array}{l}\text { Función de } \\
\text { producción Cobb- } \\
\text { Douglas }\end{array}$ & $\begin{array}{l}\text { Datos panel } \\
\text { (1984.1-1990.2) }\end{array}$ & $\begin{array}{l}\text { Eficiencia por Terminal- } \\
\text { muelle de contenedores }\end{array}$ & Decrecientes \\
\hline Tongzon (1993) & $\begin{array}{c}\text { Función de } \\
\text { producción Cobb- } \\
\text { Douglas }\end{array}$ & $\begin{array}{c}\text { Datos panel } \\
\text { (1984.1-1990.2) }\end{array}$ & $\begin{array}{l}\text { Eficiencia por Terminal- } \\
\text { muelle de contenedores }\end{array}$ & Decrecientes \\
\hline $\begin{array}{l}\text { Martínez-Budría } \\
\text { (I996) }\end{array}$ & $\begin{array}{l}\text { Función de costes } \\
\text { Cobb-Douglas }\end{array}$ & $\begin{array}{l}\text { Datos panel } \\
(1985-1989) \\
\end{array}$ & $\begin{array}{l}\text { Elasticidades coste de los } \\
\text { inputs }\end{array}$ & Crecientes \\
\hline $\begin{array}{l}\text { Jara-Díaz et al. } \\
\text { (1997) }\end{array}$ & $\begin{array}{l}\text { Función de costes } \\
\text { cuadrática }\end{array}$ & $\begin{array}{l}\text { Datos panel } \\
\text { (1985-1989) }\end{array}$ & $\begin{array}{l}\text { Costes marginales y } \\
\text { economías de diversidad }\end{array}$ & Crecientes \\
\hline $\begin{array}{l}\text { Martínez-Budría } \\
\text { et al. (1998) }\end{array}$ & $\begin{array}{l}\text { Función de costes } \\
\text { cuadrática }\end{array}$ & $\begin{array}{l}\text { Datos panel } \\
\text { (1990-1996) }\end{array}$ & $\begin{array}{l}\text { Costes Marginales, } \\
\text { elasticidades costes y PTF }\end{array}$ & Crecientes \\
\hline $\begin{array}{l}\text { Baños-Pino et al. } \\
\text { (1999) }\end{array}$ & $\begin{array}{l}\text { Función de } \\
\text { producción } \\
\text { distancia }\end{array}$ & $\begin{array}{l}\text { Datos panel } \\
\text { (1986-1995) }\end{array}$ & $\begin{array}{l}\text { Eficiencia asignativa y } \\
\text { económica }\end{array}$ & Crecientes \\
\hline $\begin{array}{l}\text { Coto-Millán et al. } \\
\qquad(2000)\end{array}$ & $\begin{array}{l}\text { Función de } \\
\text { producción } \\
\text { translog }\end{array}$ & $\begin{array}{l}\text { Datos panel } \\
\text { (1985-1989) }\end{array}$ & $\begin{array}{c}\text { Productividad y Eficiencia } \\
\text { técnica }\end{array}$ & Crecientes \\
\hline $\begin{array}{l}\text { Jara-Díaz et } \\
\text { al.(2002) }\end{array}$ & $\begin{array}{l}\text { Función de costes } \\
\text { cuadrática }\end{array}$ & $\begin{array}{l}\text { Datos panel } \\
(1986-1995)\end{array}$ & $\begin{array}{l}\text { Costes Marginales y } \\
\text { economías de diversidad }\end{array}$ & Crecientes \\
\hline Tovar (2002) & $\begin{array}{l}\text { Función de costes } \\
\text { cuadrática }\end{array}$ & $\begin{array}{l}\text { Pool de datos } \\
(1990-1999)\end{array}$ & $\begin{array}{c}\text { Costes Marginales por } \\
\text { Terminal y Economías de } \\
\text { diversidad }\end{array}$ & Crecientes \\
\hline $\begin{array}{l}\text { Cullinane et al. } \\
(2002)\end{array}$ & $\begin{array}{l}\text { Función de } \\
\text { producción }\end{array}$ & Datos panel & $\begin{array}{c}\text { Eficiencia de terminales de } \\
\text { contenedores }\end{array}$ & Crecientes \\
\hline $\begin{array}{l}\text { Cullinane et al. } \\
\text { (2006) }\end{array}$ & $\begin{array}{l}\text { Función de } \\
\text { producción }\end{array}$ & Datos panel & $\begin{array}{c}\text { Eficiencia técnica de puertos } \\
\text { de contendores }\end{array}$ & Crecientes \\
\hline $\begin{array}{l}\text { Díaz-Hernández } \\
\text { et al. (2008) }\end{array}$ & $\begin{array}{l}\text { Función de } \\
\text { producción }\end{array}$ & Datos panel & $\begin{array}{c}\text { Eficiencia en las empresas } \\
\text { estibadoras }\end{array}$ & Crecientes \\
\hline $\begin{array}{l}\text { Coto-Millán } \\
(2010)\end{array}$ & $\begin{array}{l}\text { Función de } \\
\text { producción } \\
\text { translog }\end{array}$ & $\begin{array}{l}\text { Datos panel } \\
\text { (1986-2005) }\end{array}$ & $\begin{array}{c}\text { Costes Marginales, } \\
\text { Elasticidades y Efecto } \\
\text { Averch-Jonshon (1962) }\end{array}$ & Crecientes \\
\hline $\begin{array}{l}\text { Nuñez-Sánchez } \\
\text { y Coto-Millán } \\
(2010)\end{array}$ & $\begin{array}{c}\text { Función de } \\
\text { producción } \\
\text { distancia }\end{array}$ & $\begin{array}{l}\text { Datos panel } \\
(1986-2005)\end{array}$ & $\begin{array}{c}\text { Productividades de los } \\
\text { puertos }\end{array}$ & Crecientes \\
\hline $\begin{array}{l}\text { Nuñez-Sánchez et } \\
\text { al. }(201 \mathrm{I})\end{array}$ & $\begin{array}{c}\text { Función de costes } \\
\text { translog }\end{array}$ & $\begin{array}{l}\text { Datos panel } \\
\text { (1986-2005) }\end{array}$ & $\begin{array}{c}\text { Costes Marginales, } \\
\text { Economías de diversidad y } \\
\text { Precios Ramsey }\end{array}$ & Crecientes \\
\hline $\begin{array}{l}\text { Hidalgo et al. } \\
\qquad(201 \mathrm{I})\end{array}$ & $\begin{array}{l}\text { Función de } \\
\text { producción } \\
\text { distancia }\end{array}$ & $\begin{array}{l}\text { Datos panel } \\
\text { (1986-2007) }\end{array}$ & $\begin{array}{c}\text { Eficiencia asignativa y } \\
\text { elasticidades de sustitución } \\
\text { de Morishima }\end{array}$ & Crecientes \\
\hline
\end{tabular}


Para el caso español, cabe reseñar el trabajo de Coto Millán et al. (2000), en el que se realiza un primer estudio de eficiencia técnica, a través tanto de funciones de costes (translog) como a partir de funciones de producción, para el periodo 1985-1989. Sus resultados ponen de manifiesto que los puertos de mayor tamaño son los más ineficientes desde un punto de vista económico, así como la falta de progreso tecnológico en el sistema portuario español. A subrayar, asimismo, el estudio de Baños-Pino et al. (1999) en el que se contrasta la existencia de progreso técnico en el período 1985-1995. Los resultados de las estimaciones proporcionan unos valores para el parámetro del progreso técnico que no son significativamente distintos de cero, lo que sugiere que los puertos no habrían experimentado progreso técnico exógeno en el período analizado. Algo parecido ocurre en muchos sectores manufactureros de la industria española. En ese mismo estudio la eficiencia técnica media detectada es de 0,4063 , que puede interpretarse como que, en promedio, el sector portuario español podría haber aumentado su producción un 59,36\% entre los años 1985 y 1995. Esto es, su nivel medio de eficiencia fue bajo, aunque justo es señalar que el mismo presenta una heterogeneidad muy elevada, con una horquilla que oscila entre 0,04 y 1.

\subsubsection{Método basado en el impacto económico}

Este método se basa en estimar el impacto económico portuario sobre un determinado espacio económico. De acuerdo con el mismo un puerto será tanto más competitivo cuanto mayor sea su impacto económico total (directo, indirecto e inducido) sobre el área de referencia. El puerto con más impacto tendrá una ventaja competitiva desde el punto de vista de la demanda, ya que contará con más clientes, proveedores y usuarios que otros puertos y será menos sensible, por lo tanto, a los descensos coyunturales de la demanda. Por otro lado, el puerto con más impacto económico tendrá, asimismo, una ventaja competitiva desde el punto de vista de la oferta, ya que será más difícil que los ciudadanos se opongan, por razones ecológicas y medioambientales, a ampliaciones o mejoras en sus infraestructuras. Además, el puerto con un mayor impacto atraerá más inversiones que otros puertos, lo que le otorga otro elemento de competitividad. Lógicamente, todo lo dicho será cierto únicamente en condiciones «ceteris paribus».

Los estudios clásicos de este enfoque son los realizados por Waters (1977) y Yochum y Agarwal (1987). En España también se han realizado numerosos estudios sobre el particular, acerca de cuyos resultados el artículo de Castillo-Manzano et al. (2004) ofrece una panorámica comparativa muy interesante. Entre los primeros estudios hay que citar los de Fraga y Seijas (1992) para el puerto de El Ferrol y el realizado por De Rus et al. (1994) para el puerto de La Luz y Las Palmas. Posteriormente hay que mencionar los estudios basados en tablas input-output, promovidos por Puertos del Estado, realizados por la consultora Tema (1994a, 1994b, 1995) y aplicados a los puertos gallegos. Las aplicaciones de Tema se basan en el «KIT» desarrollado por la administración americana (véanse US Maritime Administration, 1979, 1982 y 1995 como trabajos más destacados). Para el puerto de Santander, Villaverde y Coto-Millán (1995) realizaron un estudio basado en las tablas input-output aplicando el modelo de impacto económico con el que Beham (1988) había analizado el puerto de Dublín. Posteriormente a la difusión de los trabajos de Tema antes señalados, Villaverde y Coto-Millán (1996, 1998a, b) y Coto-Millán et al. (2001, 2008) realizaron otros estudios de impacto económico aplicados al puerto de 
Santander siguiendo, con adaptaciones, la metodología de Puertos del Estado; con esta misma metodología, bien que con referencia a los puertos asturianos de Avilés y Gijón, se puede contabilizar, igualmente, el trabajo de Villaverde et al. (2004). Para concluir, es interesante mencionar que en los estudios de Mateo (2010), Mateo et al. (2012) y Coto-Millán et al. (2008) se efectúa una estimación, creemos que por primera vez para un puerto español, del impacto del mismo en la ciudad en la que se ubica, en la región en la que está situada el mismo y en su hinterland.

\subsubsection{Método basado en las matrices de competitividad}

Este enfoque pretende abordar el estudio de la competitividad mediante la construcción de matrices que recogen todo un abanico de factores determinantes de la misma. El trabajo de Porter (1990), que se puede considerar como el pionero, define la competitividad sobre la base de las ventajas competitivas de las empresas a través de lo que denomina un «diamante extendido» que, a su vez, define en función de las condiciones de demanda y de oferta, y la estructura de las propias empresas y su entorno, así como de las relaciones de cada empresa con sus proveedores y clientes.

Haezendonck y Notteboom (2002) aplicaron el enfoque del diamante extendido de Porter sobre las ventajas competitivas de las naciones al caso del puerto de Amberes. Los determinantes del diamante extendido de Porter aplicados al puerto de Amberes por Haezendonck y Notteboom (2002) y por Huybrechts y Meersman (2002) son cinco: I) recursos o condiciones de factor, II) condiciones de demanda, III) industrias de soporte y relacionadas, Iv) estrategia corporativa y rivalidad, denominada también competencia, $\mathrm{y}, \mathrm{v}$ ) las condiciones de intervención del gobierno de la propia autoridad portuaria, de los gobiernos local, regional, nacional y supranacional.

Los cinco determinantes anteriores se ordenan en el eje vertical de la matriz de competitividad. En el eje horizontal de la misma se colocan las actividades que se realizan en el puerto. En el caso del puerto de Amberes en el eje horizontal de la matriz de competitividad se incluyeronn las siguientes actividades: actividades de accesibilidad marítima (ACCES), de shipping (SHIP), transhipment (LOAD), ware-housing (WARE), de valor añadido logístico (VAL), de manufacturación (MANU), de actividades de los agentes de shipping, forwarders (EXP), de distribución dentro del cluster (DISTR), y de relación con su hinterland por carretera (ROAD), por ferrocarril (RAIL) y por vías fluviales (INAV).

Definidas ya las variables de los ejes vertical y horizontal, la matriz de competitividad para el puerto de Amberes aparece reflejada en el Cuadro 2. En dicho cuadro cada una de las celdas muestra, a priori, un valor comprendido entre +2 y -2, dependiendo de la percepción positiva o negativa de esta variable para el puerto en relación a otros puertos de su mismo rango. El valor o significaría que la variable examinada no otorga ninguna ventaja ni desventaja comparativa para el puerto en cuestión.

Con un enfoque relativamente similar al desarrollado por Haezendonck y Notteboom (2002); esto es, utilizando la teoría de decisión multi-criterio que combina indicadores cuantitativos y cualitativos, Castillo et al. (2009) examinan la competitividad de los puertos españoles y concluyen que su posición en el ranking depende, en cierta medida, de cuáles sean los escenarios considerados. En todo caso, Bahía de Algeciras, Valencia y Barcelona se sitúan siempre como los puertos más competitivos mientras que Sevilla y Málaga lo hacen entre los menos competitivos. 
Cuadro 2

Matriz de competitividad del puerto de Amberes.

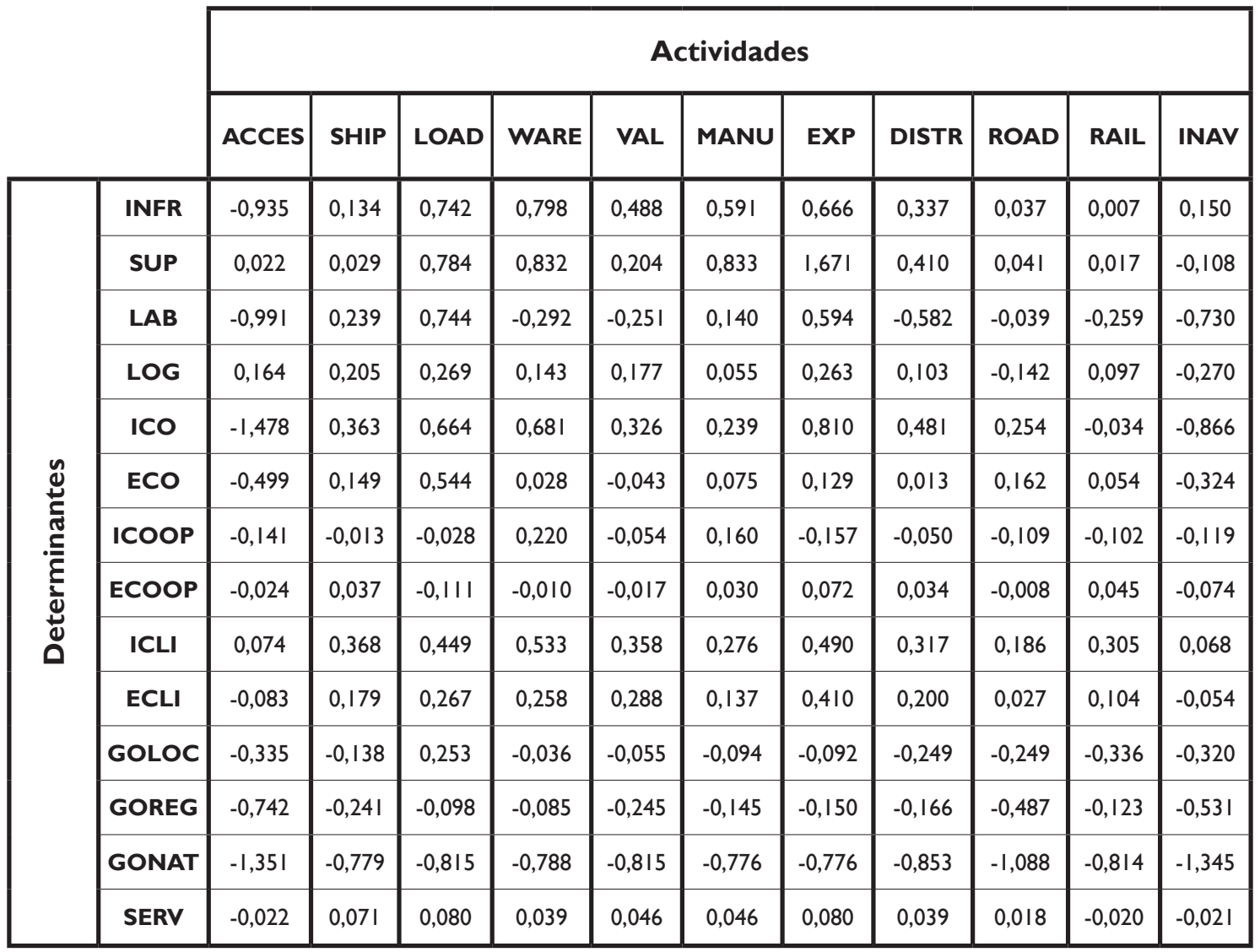

Fuente: Haezendonck y Notteboom (2002).

\subsubsection{Método basado en estudios de las desviaciones de los tráficos, en los hinterlands y forelands}

Éste método ha sido utilizado por Zubieta (1998), Vaello (1998), García (2005) y, García et al. (2007). Zubieta (1998) estudia la competencia interportuaria entre los principales puertos españoles (Bilbao, Barcelona, Valencia y Bahía de Algeciras), y entre éstos y algunos puertos europeos (Marsella, Génova, La Spezia, Livorno, Le Havre, Amberes, Rotterdam, Ámsterdam y Hamburgo). La metodología utilizada se basa en una campaña de entrevistas a operadores portuarios en los puertos españoles, completada con la aplicación del método Delfi, esto es, con una selección de importadores, exportadores, agentes de transportes, logística y comunicaciones. Posteriormente, realizan un análisis cuantitativo a partir de estadísticas procedentes de organismos oficiales.

Vaello (1998) identifica como variables fundamentales para que un determinado puerto pueda ser competitivo a las variables entorno, pues considera que si se modifican las condiciones del entorno adecuadamente se puede aumentar el volumen de tráfico captado. El estudio se refiere a los puertos peninsulares de interés general debido a que los insulares, así como los correspondientes a las ciudades de Ceuta y Melilla, se caracterizan por una cautividad natural en sus tráficos, por lo cual gozan de una ventaja 
competitiva per se. La metodología que utiliza es cuantitativa a partir del estudio de fuentes secundarias, analizando los ámbitos geográficos de los tráficos según su tonelaje, a los que clasifica en cabotaje convencional, tráfico transoceánico y flujo continental. Para finalizar, Vaello (1998) realiza una clasificación en función de la forma de presentación de la mercancía, distinguiendo los habituales tráficos de graneles líquidos, graneles sólidos y mercancía general.

García (2005) y García et al. (2007) han estudiado la competencia interportuaria en España a partir de los datos suministrados por la Dirección General de Aduanas sobre importaciones y exportaciones por puerto, provincia y tipo de mercancía para el periodo 1995-2003. Considerando que algunos de los indicadores utilizados en tales trabajos son relevantes para nuestro estudio, en la siguiente sección se hace referencia a ellos, además de proponer un nuevo indicador que trata de aproximar el nivel de competitividad de un puerto en función de los resultados obtenidos.

\section{Competencia y competitividad entre las fachadas marítimas españolas: indicadores}

La revisión de la literatura realizada en la sección anterior ha puesto de relieve la dificultad intrínseca existente para evaluar el grado de competencia entre puertos (o fachadas portuarias, en nuestro caso) y su competitividad. Por ese motivo, en esta sección tratamos de reflejar, de forma indirecta, las mencionadas competencia y competitividad portuarias, para lo cual optamos por computar tres indicadores que, a modo de preferencias reveladas por parte de los usuarios de los puertos, nos ofrecen información, fácilmente comprensible, sobre el grado de atractivo de las fachadas y, por lo tanto, sobre su mayor o menor grado de competitividad. Como ya se indicó en la Introducción, en todos los casos se hace uso de una Base de Datos Integrada de Puertos y Aduanas, referida al año 2007 y proporcionada por Puertos del Estado.

El primero de tales indicadores, que examina la procedencia provincial del tráfico de cada fachada, es el denominado «coeficiente de localización» $\left(C L_{f}\right)$, que, aplicado a nuestro caso, viene definido por la expresión:

$$
C L_{f}=1 / 2 \sum_{i}\left|\left(T_{i f} / T_{f}\right)-\left(T_{i} / T\right)\right|(1)
$$

donde $T$ es el volumen de tráfico todas las fachadas, $T_{i}$ y $T_{f}$ representan, respectivamente, los tráficos generados por la provincia $i$ y los tráficos canalizados a través de los puertos de la fachada $f$, y donde $T_{i f}$ mide el tráfico de la provincia $i$ canalizado por la fachada marítima $f$. Los valores de este coeficiente oscilan entre o y 1 , siendo el primero representativo de una situación en la que todas las provincias generan el mismo tráfico para la fachada $f$, mientras que el valor unitario del coeficiente refleja una situación en la que una sola provincia aglutina todo el tráfico de la misma.

La aplicación del coeficiente de localización al tráfico de las fachadas marítimas españolas registrado en el año 2007 da lugar al Cuadro 3, a partir del cual se pueden extraer siguientes conclusiones: 
- A nivel agregado

Todas las fachadas marítimas registran un coeficiente de localización relativamente elevado. Dicho con otras palabras, se produce una importante concentración espacial en la generación de tráfico de cada una de las fachadas consideradas. Este fenómeno, como no podía ser de otra forma, es bastante más acusado en el caso de los puertos de las dos fachadas insulares que en los de las fachadas peninsulares, siendo la fachada mediterránea la que exhibe un menor grado de concentración; asimismo, se observa que la concentración es sistemáticamente mayor en términos de peso que de valor. La concentración por modo de presentación de la mercancía varía en función de la fachada que se considere, por lo que no es sencillo generalizar, más allá del hecho evidente de que la concentración geográfica en graneles líquidos es, en todas las fachadas, mayor que la del tráfico total y que la del resto de formas de presentación.

\section{Cuadro 3}

Coeficientes de localización.

\begin{tabular}{|c|c|c|c|c|c|c|c|c|}
\hline Fachadas & Gallega & Norte & Cataluña & Levante & Surmed. & Suratl. & Canarias & Baleares \\
\hline \multicolumn{9}{|c|}{ Contenedores } \\
\hline T (valor) & 0,74 & 0,60 & 0,46 & 0,56 & 0,41 & $\mathrm{I}, 5 \mathrm{pt}$ & 0,92 & 0,99 \\
\hline$T$ (peso) & 0,75 & 0,59 & 0,57 & 0,45 & 0,45 & 0,69 & 0,93 & 0,97 \\
\hline X (valor) & 0,70 & 0,58 & 0,48 & 0,53 & 0,38 & 0,68 & 0,90 & - \\
\hline$X$ (peso) & 0,69 & 0,58 & 0,59 & 0,43 & 0,47 & 0,72 & 0,93 & - \\
\hline M (valor) & 0,77 & 0,63 & 0,44 & 0,58 & 0,52 & $0,7 \mathrm{I}$ & 0,91 & 0,99 \\
\hline$M$ (peso) & 0,80 & 0,63 & 0,55 & 0,48 & 0,43 & 0,67 & 0,90 & 0,96 \\
\hline \multicolumn{9}{|c|}{ Graneles líquidos } \\
\hline T (valor) & 0,84 & 0,75 & 0,70 & 0,71 & 0,73 & 0,86 & 0,92 & 0,91 \\
\hline$T$ (peso) & 0,85 & 0,75 & 0,73 & 0,72 & 0,74 & 0,86 & 0,92 & 0,90 \\
\hline X (valor) & 0,90 & 0,77 & 0,73 & 0,80 & 0,67 & 0,87 & 0,91 & - \\
\hline$X$ (peso) & 0,91 & 0,72 & 0,76 & 0,84 & 0,70 & 0,89 & 0,91 & - \\
\hline M (valor) & 0,84 & 0,75 & 0,70 & 0,70 & 0,75 & 0,86 & 0,92 & 0,90 \\
\hline$M$ (peso) & 0,84 & 0,75 & 0,72 & 0,71 & 0,74 & 0,85 & 0,92 & 0,90 \\
\hline \multicolumn{9}{|c|}{ Graneles sólidos } \\
\hline T (valor) & 0,57 & 0,51 & 0,73 & 0,70 & 0,77 & 0,63 & 0,98 & 0,95 \\
\hline$T$ (peso) & 0,69 & 0,58 & 0,78 & 0,72 & 0,75 & 0,76 & 0,97 & 0,95 \\
\hline X (valor) & 0,47 & 0,54 & 0,71 & 0,78 & 0,73 & 0,70 & 1,00 & 1,00 \\
\hline$X$ (peso) & 0,57 & 0,56 & 0,75 & 0,74 & 0,73 & 0,75 & 1,00 & 1,00 \\
\hline M (valor) & 0,61 & 0,51 & 0,75 & 0,69 & 0,77 & 0,63 & 0,98 & 0,95 \\
\hline M (peso) & 0,73 & 0,58 & 0,79 & 0,72 & 0,75 & 0,76 & 0,97 & 0,95 \\
\hline \multicolumn{9}{|c|}{ Mercancía general } \\
\hline $\mathbf{T}$ (valor) & 0,70 & 0,46 & 0,55 & 0,54 & 0,45 & 0,57 & 0,90 & 0,83 \\
\hline$T$ (peso) & 0,74 & 0,51 & 0,62 & 0,59 & 0,53 & 0,73 & 0,93 & 0,76 \\
\hline X (valor) & 0,67 & 0,42 & 0,55 & 0,52 & 0,54 & 0,63 & 0,93 & 0,78 \\
\hline $\mathrm{X}$ (peso) & 0,67 & 0,51 & 0,66 & 0,60 & 0,60 & 0,79 & 0,94 & 0,68 \\
\hline M (valor) & 0,75 & 0,54 & 0,64 & 0,58 & 0,47 & 0,57 & 0,87 & 0,89 \\
\hline$M$ (peso) & 0,79 & 0,53 & 0,67 & 0,60 & 0,52 & 0,71 & 0,92 & 0,96 \\
\hline \multicolumn{9}{|c|}{ Total } \\
\hline T (valor) & 0,72 & 0,57 & 0,55 & 0,57 & 0,47 & 0,68 & 0,92 & 0,86 \\
\hline $\mathrm{T}$ (peso) & 0,75 & 0,64 & 0,69 & 0,66 & 0,67 & 0,76 & 0,94 & 0,89 \\
\hline X (valor) & 0,69 & 0,51 & 0,50 & 0,54 & 0,42 & 0,67 & 0,92 & 0,79 \\
\hline X (peso) & 0,68 & 0,61 & 0,66 & 0,61 & 0,61 & 0,75 & 0,93 & 0,79 \\
\hline M (valor) & 0,74 & 0,61 & 0,58 & 0,59 & 0,54 & 0,69 & 0,91 & 0,89 \\
\hline$M$ (peso) & 0,76 & 0,65 & 0,71 & 0,68 & 0,69 & 0,76 & 0,94 & 0,90 \\
\hline
\end{tabular}

Nota: $\mathrm{T}=$ Total $; \mathrm{X}=$ Exportaciones; $\mathrm{M}=$ Importaciones 


\section{- Por exportaciones}

La concentración geográfica del tráfico de exportaciones por provincias de origen sigue una pauta similar a la del total, aunque, como norma, a un nivel más reducido. Los puertos de las fachadas insulares siguen siendo los que anotan los coeficientes más elevados, mientras que la fachada mediterránea es la que registra una mayor diversificación provincial. Por último, hay que anotar también que los graneles líquidos anotan un grado de concentración geográfica superior al del resto de formas de presentación de la mercancía.

\section{- Por importaciones}

Los resultados en este terreno son, como es lógico, similares a los expuestos en los dos puntos anteriores, siendo la nota más destacada que es en este ámbito cuando todos los coeficientes registran sistemáticamente, al menos en lo que se refiere al tráfico total, los valores más elevados; esto significa, claro está, que el tráfico de importación por provincia de destino está más concentrado que el de exportaciones por provincia de origen $\mathrm{y}$, naturalmente, que el total.

El segundo de los indicadores utilizados, que examina el tráfico desde la perspectiva provincial, es el denominado «coeficiente de especialización», que viene definido por la expresión siguiente:

$$
C E_{i}=1 / 2 \sum_{f}\left|\left(T_{i f} / T_{i}\right)-\left(T_{f} / T\right)\right|(2)
$$

donde todas las variables tienen el mismo significado que en la expresión anterior. Este coeficiente refleja en qué medida una determinada provincia utiliza, para sus tráficos, una o varias fachadas; en este sentido, y al igual que el coeficiente de localización, su valor oscila entre o y 1 , el primero de los dígitos ilustrativo de que la provincia considerada distribuye homogéneamente su tráfico entre todas las fachadas, y el segundo de que una sola fachada concentra todo el tráfico. Los resultados obtenidos al calcular este coeficiente con los datos de los flujos de tráfico portuario del 2007 (Cuadro 4) permiten, siempre dentro de una variabilidad importante, establecer las conclusiones siguientes:

\section{- A nivel agregado}

El rasgo más ilustrativo de todos es que, como norma y tal y como sugiere la lógica más elemental, las provincias que forman parte de alguna fachada marítima (es decir, que cuentan con algún puerto en alguna de las fachadas consideradas) son las que muestran un coeficiente de especialización más elevado. Siendo esto así, hay tres características adicionales que conviene destacar:

a. Que hay un grupo de provincias que no forman parte de ninguna fachada y que registran un coeficiente de especialización bastante elevado; esto es especialmente cierto en los casos de Badajoz y Ourense, pero también lo es en los de Álava, Cuenca y Huesca.

b. Que el coeficiente de especialización anota valores reducidos en algunas provincias que forman parte de alguna de las fachadas analizadas. El caso de Barcelona es el más sobresaliente dada la relevancia de la fachada catalana, pero también lo son, aunque en menor medida, los de las tres provincias insulares y Cantabria.

c. Que las provincias que muestran una mayor diversificación por fachadas son Zaragoza, en términos de valor, y Madrid en lo que concierne al peso de la mercancía transportada. 


\section{- Por exportaciones}

El rasgo más destacado es que, en promedio, los coeficientes son menos elevados que para el tráfico total, pero con una dispersión por provincias muy similar.

- Por importaciones

El coeficiente de especialización en el capítulo de las importaciones anota valores un tanto por encima de la media total pero con una dispersión provincial más reducida.

\section{- Por modos de presentación de la mercancía}

Sin incurrir en un análisis detallado de lo que ocurre en cada una de las provincias, pues sería excesivamente prolijo y difuminaría completamente la comprensión de lo sucedido, cabe destacar que el tráfico de graneles es el que registra valores del coeficiente más elevados; como norma, estos son más altos, y muestran una menor dispersión, en el caso de los graneles líquidos que en el de los sólidos. El ámbito en el que la concentración por fachadas es menor es el relativo al tráfico de contenedores, aunque aquí las disparidades provinciales son, en contrapartidas, las más abultadas.

\section{Cuadro 4}

\section{Coeficientes de especialización.}

\begin{tabular}{|c|c|c|c|c|c|c|c|c|c|c|c|c|c|c|c|c|c|c|c|c|c|c|c|c|c|c|c|c|c|c|}
\hline \multirow{2}{*}{ Provincias } & \multicolumn{6}{|c|}{ Contenedores } & \multicolumn{6}{|c|}{ Graneles líquidos } & \multicolumn{6}{|c|}{ Graneles sólidos } & \multicolumn{6}{|c|}{ Mercancía general } & \multicolumn{6}{|c|}{ Total } \\
\hline & $\mathrm{T}(\mathrm{V})$ & $T(P)$ & $x(v)$ & $\mathbf{X}(\mathbf{P})$ & $M(V)$ & $M(P)$ & $\mathrm{T}(\mathrm{V})$ & $T(P)$ & $x(v)$ & $X(P)$ & $M(v)$ & $M(P)$ & $T(V)$ & $T(P)$ & $x(v)$ & $\mathbf{x}(\mathbf{P})$ & $M(V)$ & $M(P)$ & $T(V)$ & $T(P)$ & $x(v)$ & $x(P)$ & $M(V)$ & $M(P)$ & $T(V)$ & $\mathbf{T}(\mathbf{P})$ & $x(v)$ & $x(P)$ & $M(V)$ & $M(P)$ \\
\hline A Coruña & 58 & 64 & 0,56 & 0,69 &, 62 & 58 & 0,80 & 79 & 0,93 & 0,92 & 79 & 0,79 & 85 & 0,84 & 64 & 0,71 & 0,87 & 0,85 & 0,67 & 0,75 & 0,70 & 0,56 & 0,66 & 0,85 & 0,75 & 0,81 & 0,73 & 0,78 & 0,76 & 0,81 \\
\hline Álava & 0,59 & 0,57 & 0,52 & 0,59 & 0,66 & 0,56 & 0,54 & 0,68 & 0,87 & 0,89 & 0,74 & 0,73 & 0,66 & 0,67 & 0,82 & 0,86 & 0,67 & 0,68 & 0,58 & 0,61 & 0,58 & 0,52 & 0,68 & 0,63 & 0,57 & 0,63 & 0,50 & 0,51 & 0,66 & 0,68 \\
\hline Albacete & 0,49 & 0,43 & 0,38 & 0,33 & 0,64 & 0,55 & 0,79 & 0,78 & 0,87 & 0,89 & 0,91 & 0,90 & 0,68 & 0,57 & 0,68 & 0,68 & 0,72 & 0,66 & 0,53 & 0,67 & 0,51 & 0,70 & 0,69 & 0,70 & 0,40 & 0,67 & 0,40 & 0,64 & 0,67 & 0,68 \\
\hline Alicante & 0,61 & 0,49 & 0,47 & 0,44 & 0,68 & 0,55 & 0,79 & 0,77 & 0,74 & 0,75 & 0,77 & 0,76 & 0,63 & 0,70 & 0,57 & 0,55 & 0,68 & 0,72 & 0,56 & 0,63 & 0,63 & 0,63 & 0,52 & 0,61 & 0,60 & 0,63 & 0,49 & 0,59 & 0,67 & 0,65 \\
\hline Almería & 0,56 & 0,42 & 0,54 & 0,37 & 0,63 & 0,50 & 0,83 & 0,85 & - & - & 0,84 & 0,86 & 0,88 & 0,90 & 0,90 & 0,85 & 0,87 & 0,91 & 0,75 & 0,77 & 0,82 & 0,76 & 0,69 & 0,77 & 0,62 & 0,83 & 0,57 & 0,72 & 0,67 & 0,85 \\
\hline Asturias & 0,61 & 0,69 & 0,66 & 0,72 & 0,56 & 0,65 & 0,68 & 0,58 & 0,62 & 0,60 & 0,70 & 0,57 & 0,65 & 0,64 & 0,60 & 0,63 & 0,65 & 0,64 & 0,64 & 0,63 & 0,65 & 0,62 & 0,64 & 0,65 & 0,71 & 0,71 & 0,69 & 0,70 & 0,72 & 0,71 \\
\hline Ávila & 0,21 & 0,23 & 28 & 0,19 & 0,23 & 0,42 & - & - & - & - & - & - & 7 & 0,56 & - & - & 0,67 & 0,68 & 0,59 & 0,74 & 0,68 & 0,75 & 0,79 & 0,89 & 0,43 & 0,36 & 0,53 & 0,57 & 0,72 & 0,53 \\
\hline Badajoz & 0,47 & 0,39 & 0,25 & 0,29 & 0,58 & 0,50 & 0,89 & 0,88 & 0,87 & 0,89 & 0,89 & 0,88 & 0,81 & 0,87 & 0,91 & 0,91 & 0,81 & 0,87 & 0,77 & 0,90 & 0,74 & 0,58 & 0,78 & 0,91 & 0,61 & 0,80 & 0,39 & 0,35 & 0,65 & 0,83 \\
\hline Baleares & 0,15 & 0,25 & 0,32 & 0,40 & 0,11 & 0,21 & 0,86 & 0,82 & 0,69 & 0,72 & 0,85 & 0,83 & 0,80 & 0,82 & 1,00 & 1,00 & 0,80 & 0,81 & 0,49 & 0,34 & 0,51 & 0,42 & 0,55 & 0,58 & 0,47 & 0,63 & 0,34 & 0,43 & 0,52 & 0,66 \\
\hline Barcelona & 0,48 & 0,57 & 0,50 & 0,57 & 0,45 & 0,56 & 0,60 & 0,62 & 0,71 & 0,75 &, 59 & 0,61 & 42 & 0,41 & 0,56 & 0,73 & 0,40 & 0,39 & 0,42 & 0,53 & 0,39 & 0,47 & 0,52 & 0,58 & 0,53 & 0,56 & 0,47 & 0,58 & 0,56 & 0,56 \\
\hline Burgos & 0,48 & 0,44 & 0,36 & 0,43 & 0,60 & 0,52 & 0,79 & 0 & 0,87 & 0,89 & 0,83 & 0,82 & 36 & 0,31 & 0,32 & 0,47 & 0,54 & 0,40 & 0,43 & 0,42 & 0,68 & 0,52 & 0,51 & 0,44 & 0,39 & 0,45 & 0,31 & 0,38 & 0,53 & 0,56 \\
\hline Cáceres & 0,24 & 0,34 & 0,32 & 0,51 & 0,19 & 0,32 & . & - & . & - & - & . & . & - & - & - & - & - & 0,66 & 0,57 & 0,70 & 0,61 & 0,63 & 0,60 & 0,23 & 0,33 & 0,35 & 0,44 & 0,19 & 0,38 \\
\hline Cádiz & 0,78 & 0,82 & 0,74 & 0,82 & 0,75 & 0,78 & 0,82 & 0,83 & 0,73 & 0,74 & 0,83 & 0,84 & 0,68 & 0,78 & 0,76 & 0,82 & 0,69 & 0,79 & 0,55 & 0,65 & 0,69 & 0,77 & 0,49 & 0,65 & 0,74 & 0,77 & 0,72 & 0,76 & 0,74 & 0,77 \\
\hline Cantabria & 0,60 & 0,64 & 0 & 0,70 & 0,62 & 0 & 0 & 0 & 0,1 & 7 & 0,80 & 0 & 3 & 0 & 62 & 0,63 & 0,63 & 65 & & 0,47 & 0,60 & 0,66 & 0,63 & 0,48 & 0,67 & 0,57 & 0,60 & 0,71 & ,71 & 0,53 \\
\hline Castellón & 0,56 & 0,53 & 0,56 & 0,4 & 0,63 & 0 & 0,71 & 0,74 & 0,8 & 0,86 & 69 & 0,72 & 61 & 0,79 & 0,41 & 0,78 & 0,67 & 0,79 & 0,37 & 0,59 & 0,44 & 0,61 & 0,57 & 0,63 & 0,61 & 0,72 & 0,58 & 0,69 & 0,66 & 0,73 \\
\hline $\begin{array}{c}\text { Ciudad } \\
\text { Real }\end{array}$ & 0,49 & 0,28 & 0,36 & 0,21 & 0,67 & 0,56 & 0,50 & 0,52 & 0,62 & 0,67 & 0,56 & 0,54 & 0,89 & 0,86 & 0,82 & 0,86 & 0,90 & 0,86 & 0,63 & 0,61 & 0,67 & 0,61 & 0,62 & 0,69 & 0,29 & 0,45 & 0,25 & 0,42 & 0,67 & 0,57 \\
\hline Córdoba & 0,60 & 0,54 & 0,60 & 0,69 & 0,62 & 0,51 & 0,71 & 0,71 & 0,61 & 0,62 & 0,86 & 0,87 & 0,65 & 0,73 & 0,77 & 0,83 & 0,63 & 0,73 & 0,58 & 0,52 & 0,66 & 0,66 & 0,50 & 0,49 & 0,54 & 0,53 & 0,53 & 0,55 & 0,56 & 0,53 \\
\hline Cuenca & 0,58 & 0,42 & 0,48 & 0,33 & 0,69 & 0,52 & 0,64 & 0,64 & 0,87 & 0,89 & 0,86 & 0,87 & 0,86 & 0,83 & 0,82 & 0,86 & 0,86 & 0,83 & 0,26 & 0,39 & 0,47 & 0,56 & 0,53 & 0,40 & 0,53 & 0,72 & 0,34 & 0,42 & 0,65 & 0,74 \\
\hline Girona & 0,32 & 0,51 & 0,40 & 0,45 & 0,28 & 0,56 & 0,76 & 0,78 & - & - & 0,76 & 0,78 & 0,76 & 0,73 & 0,91 & 0,91 & 0,76 & 0,73 & 0,33 & 0,47 & 0,49 & 0,54 & 0,36 & 0,48 & 0,38 & 0,42 & 0,40 & 0,45 & 0,38 & 0,43 \\
\hline Granada & 0,39 & 0,44 & 0,43 & 0,38 & 0,36 & 0,48 & 0,82 & 0,85 & 0,63 & 0,65 & 0,86 & 0,87 & 71 & 0,81 & 0,95 & 0,86 & 0,67 & 0,79 & 0,61 & 0,66 & 0,68 & 0,71 & 0,73 & 0,87 & 0,52 & 0,77 & 0,53 & 0,56 & 0,69 & 0,82 \\
\hline Guadalajara & 0,55 & 0,40 & | 0,48 & | 0,24 & 0,60 & 0,50 & - & - & - & - & - & - & 0,66 & 0,67 & 0,66 & 0,66 & 0,62 & 0,54 & 0,68 & 0,38 & 0,69 & 0,50 & 0,69 & 0,54 & 0,42 & 0,42 & 0,42 & 0,36 & 0,42 & 0,47 \\
\hline Guipúzcoa & 0,54 & 0,57 & 0,51 & 0,54 & 0,59 & 0,62 & 0,69 & 0,67 & 0,71 & 0,68 & 0,75 & 0,78 & 0,60 & 0,65 & 0,49 & 0,46 & 0,60 & 0,65 & 0,60 & 0,61 & 0,63 & 0,59 & 0,51 & 0,64 & 0,58 & 0,68 & 0,56 & 0,58 & 0,60 & 0,71 \\
\hline
\end{tabular}




\begin{tabular}{|c|c|c|c|c|c|c|c|c|c|c|c|c|c|c|c|c|c|c|c|c|c|c|c|c|c|c|c|c|c|c|}
\hline \multirow{2}{*}{ Provincias } & \multicolumn{6}{|c|}{ Contenedores } & \multicolumn{6}{|c|}{ Graneles líquidos } & \multicolumn{6}{|c|}{ Graneles sólidos } & \multicolumn{6}{|c|}{ Mercancía general } & \multicolumn{6}{|c|}{ Total } \\
\hline & $T(V)$ & $T(P)$ & $x(V)$ & $x(P)$ & $M(V)$ & $M(P)$ & $T(V)$ & $T(P)$ & $x(v)$ & $x(P)$ & $M(V)$ & $M(P)$ & $T(V)$ & $\mathbf{T}(\mathbf{P})$ & $x(v)$ & $x(P)$ & $M(V)$ & $M(P)$ & $T(V)$ & $T(P)$ & $x(v)$ & $x(P)$ & $M(v)$ & $M(P)$ & $\mathrm{T}(\mathrm{V})$ & $T(P)$ & $x(v)$ & $\mathbf{x}(\mathbf{P})$ & $M(v)$ & $M(P)$ \\
\hline Huelva & 0,70 & 0,74 & 0,75 & 0,83 & 0,47 & 0,45 & 0,89 & 0,89 & 0,89 & 0,91 & 0,89 & 0,89 & 0,64 & 0,79 & 0,59 & 0,67 & 0,65 & 0,84 & 0,77 & 0,78 & 0,87 & 0,91 & 0,58 & 0,60 & 0,84 & 0,86 & 0,80 & 0,79 & 0,85 & 0,87 \\
\hline Huesca & 0,37 & 0,53 & 0,41 & 0,56 & 0,44 & 0,47 & 0,76 & 0,78 & 0,87 & 0,89 & 0,76 & 0,78 & 0,75 & 0,84 & 0,70 & 0,83 & 0,86 & 0,86 & 0,57 & 0,75 & 0,55 & 0,74 & 0,40 & 0,42 & 0,52 & 0,72 & 0,46 & 0,65 & 0,64 & 0,80 \\
\hline Jaén & 0,55 & 0,37 & 0,48 & 0,27 & 0,59 & 0,46 & 0,71 & 0,76 & 0,63 & 0,64 & 0,86 & 0,87 & 0,86 & 0,90 & . & - & 0,85 & 0,90 & 0,62 & 0,62 & 0,68 & 0,69 & 0,56 & 0,59 & 0,57 & 0,57 & 0,58 & 0,51 & 0,57 & 0,59 \\
\hline La Rioja & 0,59 & 0,59 & 0,58 & 0,59 & 0,56 & 0,59 & 0,75 & 0,63 & 0,87 & 0,89 & 0,76 & 0,78 & 0,65 & 0,60 & 0,66 & 0,66 & 0,60 & 0,60 & 0,62 & 0,53 & 0,75 & 0,77 & 0,56 & 0,52 & 0,49 & 0,44 & 0,50 & 0,44 & 0,43 & 0,43 \\
\hline Las Palmas & 0,59 & 0,67 & 0,63 & 0,77 & 0,65 & 0,67 & 0,83 & 0,86 & 0,96 & 0,95 & 0,83 & 0,86 & 0,63 & 0,89 & 0,66 & 0,82 & 0,63 & 0,89 & 0,25 & 0,46 & 0,49 & 0,61 & 0,22 & 0,41 & 0,43 & 0,75 & 0,49 & 0,64 & 0,46 & 0,76 \\
\hline León & 0,49 & 0,31 & 0,43 & 0,42 & 0,56 & 0,45 & $\mid 0,74$ & \begin{tabular}{|l|l|} 
& 0,72 \\
\end{tabular} & - & - & 0,75 & 0,73 & 0,50 & 0,49 & 0,66 & 0,66 & 0,50 & 0,48 & 0,45 & 0,69 & 0,58 & 0,37 & 0,43 & 0,78 & 0,45 & $\mid 0,37$ & 0,44 & 0,40 & 0,46 & 0,41 \\
\hline Lleida & 0,43 & 0,66 & 0,48 & 0,71 & 0,43 & 0,56 & 0,72 & 0,65 & 0,68 & 0,71 & 0,71 & 0,64 & 0,67 & 0,83 & 0,68 & 0,87 & 0,75 & 0,84 & 0,52 & 0,57 & 0,57 & 0,61 & 0,53 & 0,49 & 0,45 & 0,70 & 0,44 & 0,71 & 0,55 & 0,65 \\
\hline Lugo & | 0,67 & $\mid 0,77$ & 0,65 & $\mid 0,81$ & 0,69 & 0,68 & & - & 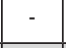 & & - & & 0,89 & 0,86 & 0,80 & 0,84 & 0,90 & 0,86 & 0,63 & 0,81 & 0,65 & 0,79 & 0,67 & 0,77 & 0,83 & 0,90 & 0,69 & 0,85 & 0,87 & 0,90 \\
\hline Madrid & 0,36 & 0,21 & 0,23 & 0,08 & 0,45 & 0,31 & 0,29 & 0,35 & 0,45 & 0,64 & 0,31 & 0,32 & 0,16 & 0,19 & 0,38 & 0,18 & 0,18 & 0,20 & 0,29 & 0,21 & 0,27 & 0,18 & 0,44 & 0,27 & 0,28 & 0,17 & 0,17 & 0,12 & 0,37 & 0,19 \\
\hline Málaga & 0,77 & 0,76 & 0,69 & 0,72 & 0,82 & 0,78 & 0,84 & 0,80 & 0,76 & 0,76 & 0,86 & 0,77 & 0,73 & 0,82 & 0,75 & 0,81 & 0,73 & 0,83 & 0,67 & 0,67 & 0,82 & 0,76 & 0,54 & 0,62 & 0,71 & 0,77 & 0,72 & 0,69 & 0,74 & 0,79 \\
\hline Murcia & 0,57 & 0,47 & 0,54 & 0,40 & 0,61 & 0,56 & 0,77 & 0,77 & 0,84 & 0,86 & 0,76 & 0,75 & 0,53 & 0,71 & 0,72 & 0,75 & 0,54 & 0,71 & 0,73 & 0,66 & 0,60 & 0,58 & 0,70 & 0,66 & 0,57 & 0,71 & 0,58 & 0,60 & 0,57 & 0,73 \\
\hline Navarra & 0,46 & 0,48 & 0,40 & 0,43 & 0,55 & 0,59 & 0,80 & 0,78 & 0,77 & 0,74 & 0,81 & 0,75 & 0,39 & 0,48 & 0,44 & 0,54 & 0,39 & 0,47 & 0,26 & 0,38 & 0,30 & 0,31 & 0,48 & 0,53 & 0,32 & 0,45 & 0,34 & 0,39 & 0,51 & 0,56 \\
\hline Ourense & 0,55 & 0,68 & | 0,54 & 0,77 & 0,68 & 0,57 & 0,93 & 0,93 & - & - & 0,93 & 0,93 & 0,67 & 0,68 & - & - & 0,67 & 0,68 & 0,84 & 0,82 & 0,82 & 0,81 & 0,85 & 0,80 & 0,70 & 0,73 & 0,77 & 0,79 & 0,70 & 0,63 \\
\hline Palencia & 0,47 & 0,21 & 0,42 & 0,24 & 0,53 & 0,19 & & - & - & & - & & 0,38 & 0,44 & & - & 0,38 & 0,44 & 0,29 & 0,54 & 0,35 & 0,47 & 0,51 & 0,77 & 0,40 & 0,23 & 0,31 & 0,30 & 0,44 & 0,32 \\
\hline Pontevedra & 0,79 & 0,81 & 0,70 & 0,75 & 0,89 & 0,89 & 0,53 & 0,88 & 0,54 & 0,67 & 0,89 & 0,91 & 0,46 & 0,74 & 0,78 & 0,84 & 0,47 & 0,74 & 0,79 & 0,79 & 0,76 & 0,76 & 0,79 & 0,81 & 0,79 & 0,81 & 0,74 & 0,77 & 0,84 & 0,84 \\
\hline Salamanca & 0,42 & 0,25 & 0,20 & 0,35 & 0,56 & 0,41 & 0,77 & 0,77 & 0,73 & 0,69 & - & & 0,82 & 0,88 & & - & 0,82 & 0,88 & 0,46 & 0,32 & 0,47 & 0,54 & 0,48 & 0,53 & 0,54 & 0,51 & 0,67 & 0,59 & 0,44 & 0,52 \\
\hline Tenerife & 0,56 & 0,62 & 0,73 & 0,77 & 0,60 & 0,60 & 0,89 & 0,93 & 0,95 & 0,94 & 0,88 & 0,93 & 0,80 & 0,91 & 0,73 & 0,69 & 0,80 & 0,91 & 0,36 & 0,36 & 0,53 & 0,44 & 0,28 & 0,40 & 0,51 & 0,88 & 0,39 & 0,82 & 0,55 & 0,88 \\
\hline Segovia & 0,42 & 0,36 & 0,39 & 0,22 & 0,51 & 0,49 & - & - & - & - & - & - & 0,55 & 0,51 & 0,88 & 0,86 & 0,67 & 0,68 & 0,47 & 0,45 & 0,79 & 0,72 & 0,53 & 0,45 & 0,43 & 0,50 & 0,50 & 0,48 & 0,55 & 0,53 \\
\hline Sevilla & 0,68 & 0,74 & 0,71 & 0,79 & 0,60 & 0,65 & 0,80 & 0,83 & 0,57 & 0,61 & 0,87 & 0,85 & 0,67 & 0,64 & 0,72 & 0,76 & $\mid 0,64$ & 0,62 & 0,58 & 0,77 & 0,77 & 0,77 & 0,49 & 0,77 & 0,64 & 0,67 & 0,71 & 0,72 & 0,59 & 0,65 \\
\hline Soria & 0,23 & 0,16 & 0,26 & 0,20 & 0,60 & 0 , & & - & - & & - & & - & - & & - & & & 0,40 & 0,51 & 0,49 & 0,68 & 0,68 & 0,61 & 0,27 & 0,40 & 0,32 & 0,44 & 0,57 & 0,44 \\
\hline Tarragona & 0,40 & 0,56 & 0,42 & 0,59 & 0,42 & 0,53 & 0,73 & 0,75 & 0,78 & 0,80 & 0,72 & 0,75 & 0,80 & 0,81 & 0,69 & 0,77 & 0,80 & 0,81 & $\mid 0,74$ & 0,81 & 0,70 & 0,80 & 0,78 & 0,81 & 0,64 & 0,77 & 0,63 & 0,77 & 0,64 & 0,77 \\
\hline Teruel & 0,44 & 0,35 & 0,52 & 0,36 & 0,19 & 0,36 & 0,79 & 0,78 & - & & 0,77 & 0,77 & 0,71 & 0,67 & 0,66 & 0,70 & 0,74 & 0,68 & 0,47 & 0,40 & 0,52 & 0,53 & 0,46 & 0,39 & 0,34 & 0,54 & 0,49 & 0,45 & 0,41 & 0,57 \\
\hline Toledo & 0,59 & 0,45 & 0,46 & 0,28 & 0,66 & 0,56 & 0,75 & 0,76 & \begin{tabular}{|l|l|} 
& 0,84 \\
\end{tabular} & $\mid 0,87$ & 0,76 & 0,78 & 0,54 & 0,54 & 0,90 & 0,89 & 0,67 & 0,68 & 0,49 & 0,38 & 0,65 & 0,58 & 0,54 & 0,41 & 0,58 & 0,56 & 0,47 & 0,52 & 0,62 & 0,57 \\
\hline Valencia & 0,65 & 0,50 & 0,57 & 0,43 & 0,68 & 0,57 & 0,71 & 0,75 & 0,71 & 0,65 & 0,74 & 0,75 & 0,76 & 0,80 & 0,76 & 0,83 & 0,76 & 0,79 & 0,60 & 0,67 & 0,45 & 0,58 & 0,76 & 0,68 & 0,63 & 0,70 & 0,50 & 0,60 & 0,72 & 0,73 \\
\hline Valladolid & 0,38 & 0,26 & 0,41 & 0,24 & 0,29 & 0,29 & 0,71 & 0,64 & 0,87 & 0,89 & 0,72 & 0,65 & 0,51 & 0,60 & 0,91 & 0,75 & 0,52 & 0,60 & 0,56 & 0,53 & 0,52 & 0,61 & 0,57 & 0,55 & 0,21 & 0,53 & 0,35 & 0,30 & 0,35 & 0,57 \\
\hline Vizcaya & 0,64 & 0,66 & 0,60 & 0,66 & 0,68 & 0,66 & 0,78 & 0,78 & 0,73 & 0,69 & 0,79 & 0,79 & 0,63 & 0,62 & 0,54 & 0,62 & 0,64 & 0,62 & 0,61 & 0,57 & 0,62 & 0,51 & 0,61 & 0,58 & 0,71 & 0,71 & 0,65 & 0,68 & 0,74 & 0,71 \\
\hline Zamora & 0,31 & 0,41 & 0,43 & 0,51 & 0,18 & 0,36 & - & - & - & 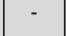 & - & - & 0,62 & 0,64 & 0,88 & 0,86 & 0,67 & 0,71 & 0,66 & 0,73 & 0,62 & 0,68 & 0,90 & 0,85 & 0,26 & 0,38 & 0,32 & 0,43 & 0,33 & 0,54 \\
\hline Zaragoza & 0,25 & 0,44 & 0,30 & 0,48 & 0,22 & 0,38 & 0,26 & 0,40 & 0,87 & 0,89 & 0,43 & 0,43 & 0,50 & 0,56 & 0,75 & 0,49 & 0,51 & 0,62 & 0,26 & 0,25 & 0,33 & 0,27 & 0,34 & 0,28 & 0,18 & 0,33 & 0,23 & 0,40 & 0,22 & 0,33 \\
\hline Media & 0,50 & 0,49 & 0,48 & 0,49 & 0,54 & 0,52 & 0,73 & 0,75 & 0,76 & 0,78 & 0,77 & 0,77 & 0,65 & 0,69 & 0,71 & 0,74 & 0,67 & 0,71 & 0,55 & 0,58 & 0,60 & 0,61 & 0,58 & 0,61 & 0,52 & 0,60 & 0,50 & 0,56 & 0,58 & 0,63 \\
\hline Desv.TíPICA & 0,15 & 0,17 & 0,14 & 0,20 & $\mid 0,17$ & 0,13 & 0,14 & 0,12 & 0,12 & 0,11 & 0,12 & 0,13 & 0,15 & 0,16 & 0,16 & 0,16 & 0,15 & 0,16 & 0,15 & 0,16 & 0,14 & 0,15 & 0,14 & 0,16 & 0,17 & 0,18 & 0,15 & 0,16 & 0,15 & 0,16 \\
\hline VARIACIÓN & 0,29 & 0,35 & 0,29 & $\mid 0,41$ & 0,32 & 0,26 & 0,19 & 0,17 & 0,16 & $\mid 0,14$ & 0,16 & 0,17 & 0,24 & 0,24 & 0,22 & 0,21 & 0,22 & 0,22 & 0,28 & 0,28 & 0,24 & 0,24 & | 0,24 & 0,26 & 0,32 & 0,29 & 0,31 & 0,29 & 0,26 & 0,26 \\
\hline
\end{tabular}

Nota: $\mathrm{T}(\mathrm{V})=$ Total $($ Valor); $\mathrm{T}(\mathrm{P})=$ Total $($ Peso); $\mathrm{X}(\mathrm{V})=$ Exportaciones (Valor); $\mathrm{X}(\mathrm{P})=$ Exportaciones (Peso); $M(\mathrm{~V})=$ Importaciones (Valor); $M(P)=$ Importaciones (Peso). 
Por último, y con la finalidad de profundizar un poco más en el conocimiento del atractivo/competitividad de cada una de las fachadas marítimas, proponemos un nuevo indicador (ATep ) que, a diferencia de los anteriores, toma en consideración la distancia como factor parcialmente explicativo del mencionado grado de atractivo/competitividad (Villaverde y Maza, 2012, demuestran la importancia del factor distancia). Expresado este índice en términos relativos (que denominamos ATRep) para facilitar su interpretación -haciendo la media nacional igual a 100-, la expresión propuesta es la siguiente:

donde

$$
\operatorname{ATRep}_{f}=\left(\text { ATep }_{f} / \text { ATep }\right) * 100
$$

$$
A T e p_{f}=\Sigma_{j} \sum_{i} T_{i j} D_{i j}
$$

$\mathrm{y}$

$$
\text { ATep }=\sum_{f} P_{f} A T e p_{f}(5)
$$

En estas expresiones, $P_{f}$ es el peso de la fachada $f$ en el conjunto del tráfico marítimo español, $T_{i j}$ mide el tráfico de la provincia $i$ a través del puerto $j$ perteneciente a la fachada considerada $f$, y $D_{i j}$ representa la distancia entre la provincia $i$ y el puerto $j$ de dicha fachada. Con objeto de analizar la robustez de los resultados a distintos conceptos de distancia, optamos por utilizar cuatro indicadores de distancia alternativos:

1. La distancia $D_{i j}$ propiamente dicha, expresada en kilómetros entre la capital de la provincia $i$ y la del puerto $j$ (indicador que denominamos D1).

2. La raíz cuadrada de $D_{i j}(\mathrm{D} 2)$. La justificación de este indicador estriba en que parece un poco discutible considerar de la misma forma, por ejemplo, 1.000 Tms. desplazadas $1 \mathrm{Km}$. que $1 \mathrm{Tm}$. desplazada $1.000 \mathrm{kms}$., circunstancia que se produce con la definición D1 pero no con la D2.

3. $D_{i j}$ dividida por la distancia media de la provincia $i$ a todos los puertos (D3). Esta medida expresa la distancia en términos relativos con respecto a la distancia media que ha de recorrer una mercancía para llegar a puerto en el conjunto del país.

4. $D_{i j}$ dividida por la distancia de la provincia $i$ al puerto más cercano (D4). Esta medida es similar a la anterior pero pondera en mayor medida las mercancías provenientes de provincias cercanas a puertos.

Cuando la provincia de referencia es la misma en la que está situado el puerto, la distancia (que en base al primer indicador sería igual a cero en todos los casos) se calcula como (Crozet, 2004):

$$
D_{i j}=0,66 \sqrt{\hat{A} r e a_{i} / \pi}
$$

Tal y como está construido el índice, valores iguales, superiores e inferiores a 100 para una fachada específica representan, respectivamente, una situación en la que tal fachada es igual, más o menos atractiva que la media; naturalmente, cuanto más se aleje el índice del valor 100 hacia arriba (hacia abajo), mayor es la diferencia positiva (negativa) en el grado de atracción de la fachada con relación a la referida media. En este sentido, los resultados obtenidos al aplicar las expresiones anteriores se muestran en el Cuadro 5, a partir de cuya consideración se pueden extraer las siguientes conclusiones de interés: 
- A nivel agregado

Tomando en consideración todo el tráfico de cada una de las fachadas, la Surmediterránea resulta ser la más atractiva en términos de valor y la Norte en términos de peso. Baleares y Canarias son, por el contrario, las fachadas menos atractivas seguidas, a cierta distancia, de la fachada Suratlántica.

- Por modo de presentación de la mercancía

La fachada Norte es la más atractiva, en valor y peso, tanto en lo que atañe a la mercancía general como a los graneles sólidos; la fachada Surmediterránea ocupa la misma posición de privilegio en lo que atañe a los graneles líquidos y Levante en lo que se refiere al tráfico de contenedores. En estos cuatro modos de tráfico las fachadas balear y canaria ocupan la últimas posiciones, repartiéndose la Suratlántica y la Gallega las siguientes plazas del furgón de cola.

- Por exportaciones e importaciones

Las dos situaciones descritas se reproducen, tanto en relación con el total como con referencia a los distintos modos de tráfico, cuando sus magnitudes agregadas se desglosan en tráfico de exportaciones y tráfico de importaciones; en particular, la reproducción es casi mimética en el caso de las importaciones, terreno que, por tener más importancia en el conjunto del tráfico, configura mejor lo sucedido a nivel agregado.

- Por medida de distancia

Aunque no hay duda de que la distancia importa respecto al atractivo que cada puerto tiene para las distintas provincias, tal y como se ha puesto de relieve en numerosos estudios, los distintos indicadores de distancia utilizados no afectan de manera sustancial al grado de atractivo de las distintas fachadas marítimas. Los resultados son, por tanto, bastante robustos en este sentido.

Por último, conviene anotar que, aunque una simple inspección del Cuadro 5 puede erróneamente darnos a entender que, en un número amplio de situaciones, los resultados obtenidos para el indicador de atractivo/competitividad varían apreciablemente según se considere el valor o el peso de las mercancías comerciadas, lo cierto es que, tal y como se muestra en el Cuadro 6, los coeficientes de correlación valor-peso en todas las manifestaciones del tráfico portuario son muy elevados. En concreto, el coeficiente más bajo es igual a 0,76 (para las importaciones de mercancía general cuando se utiliza como indicador de distancia el D4) y nada menos que en 46 de los 60 casos analizados (el $76,7 \%$ ) supera el valor de 0,9 , lo que constituye una manifestación muy clara de que el grado de atractivo de las fachadas no varía demasiado sea cual sea (valor o precio) la magnitud representativa del tráfico que se considere. Este último resultado resulta muy importante en nuestra opinión, pues incide en la robustez de las conclusiones obtenidas. 


\section{Cuadro 5}

Índices de atractivo expost.

\begin{tabular}{|c|c|c|c|c|c|c|c|c|c|c|c|c|}
\hline \multirow{2}{*}{ Fachadas } & \multicolumn{2}{|c|}{ DI } & \multicolumn{2}{|c|}{ D2 } & \multicolumn{2}{|c|}{ D3 } & \multicolumn{2}{|c|}{ D4 } & \multicolumn{2}{|c|}{ Media } & \multicolumn{2}{|c|}{ Prelación } \\
\hline & Valor & Peso & Valor & Peso & Valor & Peso & Valor & Peso & Valor & Peso & Valor & Peso \\
\hline \multicolumn{13}{|c|}{ Contenedores } \\
\hline Galicia & 27,21 & 23,57 & 19,38 & 18,25 & 22,59 & 20,09 & 45,86 & 38,55 & 28,76 & 25,12 & 5 & 5 \\
\hline Norte & 80,97 & 72,99 & 62,48 & 58,68 & 71,46 & 67,12 & 146,67 & 106,88 & 90,40 & 76,42 & 4 & 4 \\
\hline Cataluña & 102,77 & 79,86 & 123,79 & 83,79 & 101,27 & 76,62 & 93,86 & 92,61 & 105,42 & 83,22 & 3 & 2 \\
\hline Levante & 125,58 & $|46,3|$ & 120,59 & 155,86 & 137,69 & 152,59 & 89,65 & || $9,3 \mid$ & 118,38 & 143,52 & I & 1 \\
\hline Surmediterránea & 118,16 & 83,43 & 73,45 & 53,08 & 108,77 & 75,63 & 152,08 & 107,24 & 113,11 & 79,85 & 2 & 3 \\
\hline Suratlántica & 19,67 & 21,16 & $|3,0|$ & 14,96 & 16,27 & 18,00 & 41,23 & 39,40 & 22,55 & 23,38 & 6 & 6 \\
\hline Baleares & 0,00 & 0,00 & 0,00 & 0,00 & 0,00 & 0,00 & 0,00 & 0,00 & 0,00 & 0,00 & 8 & 8 \\
\hline Canarias & 3,45 & 3,43 & 3,46 & 3,54 & 3,31 & 3,08 & 3,25 & 4,08 & 3,37 & 3,53 & 7 & 7 \\
\hline \multicolumn{13}{|c|}{ Graneles líquidos } \\
\hline Galicia & 39,05 & 43,81 & 38,79 & 43,21 & 37,21 & 40,33 & 64,33 & 80,59 & 44,85 & 51,99 & 5 & 5 \\
\hline Norte & 81,22 & 79,41 & 85,47 & 88,29 & 81,02 & 75,93 & 118,70 & $13 \mid, 66$ & 91,60 & 93,82 & 4 & 4 \\
\hline Cataluña & $|22,4|$ & $|06,7|$ & 134,12 & 122,84 & 128,02 & 111,27 & 105,17 & 73,86 & 122,43 & 103,67 & 2 & 3 \\
\hline Levante & 124,73 & 134,49 & 133,32 & 143,87 & 125,04 & 144,40 & 99,34 & 92,60 & $|20,6|$ & 128,84 & 3 & 2 \\
\hline Surmediterránea & 153,23 & 169,79 & 109,15 & 109,20 & 145,67 & 155,06 & 165,43 & 201,16 & 143,37 & 158,80 & I & 1 \\
\hline Suratlántica & 33,24 & 39,93 & 47,91 & 54,43 & 34,06 & 40,84 & 25,83 & $3 \mathrm{I}, 47$ & 35,26 & 41,67 & 6 & 6 \\
\hline Baleares & 6,71 & 6,07 & 5,58 & 4,83 & 6,65 & 5,72 & 6,52 & 7,81 & 6,37 & 6,11 & 8 & 8 \\
\hline Canarias & 21,06 & 22,42 & 21,65 & 23,52 & 17,89 & $|7,9|$ & 24,40 & 30,03 & 21,25 & 23,47 & 7 & 7 \\
\hline \multicolumn{13}{|c|}{ Graneles sólidos } \\
\hline Galicia & 142,15 & 134,64 & 97,68 & 96,48 & 137,26 & 129,79 & 130,63 & 126,50 & 126,93 & 121,85 & 2 & 2 \\
\hline Norte & 183,24 & 146,10 & 175,49 & 157,97 & 185,14 & 147,20 & 182,99 & $|47,0|$ & $|8|, 7 \mid$ & 149,57 & I & I \\
\hline Cataluña & 37,69 & 44,82 & 50,70 & 58,95 & 36,34 & 43,33 & 48,12 & 50,47 & 43,21 & 49,39 & 4 & 6 \\
\hline Levante & 33,45 & 94,68 & 48,55 & 94,43 & 33,65 & 97,07 & 30,22 & 87,93 & 36,47 & 93,53 & 5 & 3 \\
\hline Surmediterránea & 30,07 & 57,88 & 43,43 & 52,21 & 31,05 & 60,38 & 34,26 & 60,77 & 34,70 & 57,81 & 6 & 5 \\
\hline Suratlántica & 62,35 & 59,10 & 76,07 & 59,60 & 62,13 & 58,00 & 61,95 & 66,62 & 65,62 & 60,83 & 3 & 4 \\
\hline Baleares & 0,28 & 0,54 & 0,67 & $\mathrm{I}, 14$ & 0,28 & 0,53 & 0,28 & 0,67 & 0,38 & 0,72 & 8 & 8 \\
\hline Canarias & 0,82 & 1,68 & 1,50 & 4,33 & 0,69 & 1,08 & 1,15 & 3,81 & 1,04 & 2,73 & 7 & 7 \\
\hline \multicolumn{13}{|c|}{ Mercancía general } \\
\hline Galicia & 38,34 & 49,86 & 45,39 & 56,68 & 38,40 & 47,83 & 45,67 & 59,55 & 41,95 & 53,48 & 5 & 5 \\
\hline Norte & 147,64 & 140,94 & 144,02 & 148,88 & 139,28 & 140,87 & 174,65 & $|4|, 82$ & $15 \mid, 40$ & 143,13 & I & I \\
\hline Cataluña & 79,30 & 107,34 & 95,24 & 92,17 & 85,01 & 102,55 & 61,72 & 133,87 & 80,32 & 108,98 & 4 & 2 \\
\hline Levante & 77,49 & 97,63 & 78,84 & 104,47 & 67,30 & 100,48 & 109,67 & 81,87 & 83,32 & 96,11 & 3 & 3 \\
\hline Surmediterránea & 155,25 & $|02,9|$ & 132,05 & 75,03 & 170,46 & 106,48 & 102,06 & 81,54 & 139,95 & 91,49 & 2 & 4 \\
\hline Suratlántica & 31,96 & 30,08 & 28,59 & $3 \mid, 84$ & 30,76 & 32,64 & 25,23 & 19,70 & 29,14 & 28,56 & 6 & 6 \\
\hline Baleares & 5,14 & 3,46 & 4,27 & 2,23 & 3,73 & 2,76 & 15,23 & 5,14 & 7,09 & 3,40 & 7 & 7 \\
\hline Canarias & $\mathrm{I}, 14$ & 2,77 & 1,66 & 3,05 & 1,23 & 2,73 & 0,69 & 2,17 & 1,18 & 2,68 & 8 & 8 \\
\hline \multicolumn{13}{|c|}{ Total } \\
\hline Galicia & 42,44 & 77,98 & 37,47 & 63,88 & 40,18 & 74,64 & 51,90 & 82,35 & 43,00 & 74,72 & 5 & 5 \\
\hline Norte & 122,67 & 123,17 & 106,08 & 126,11 & 114,00 & 121,22 & $167,8 \mid$ & 141,62 & 127,64 & 128,03 & 2 & 1 \\
\hline Cataluña & 98,51 & 88,24 & 122,32 & 97,97 & 101,65 & 87,15 & 79,31 & 94,36 & 100,45 & 91,93 & 4 & 4 \\
\hline Levante & 109,04 & 130,25 & 114,72 & 137,88 & 112,93 & 137,08 & 102,68 & 101,51 & 109,84 & 126,68 & 3 & 2 \\
\hline Surmediterránea & 141,16 & 110,27 & 102,79 & 80,72 & $|4|, 6 \mid$ & 106,15 & 127,73 & 115,86 & 128,32 & 103,25 & I & 3 \\
\hline Suratlántica & $30,4 I$ & 44,37 & 27,96 & 49,18 & 28,37 & 44,22 & 34,16 & 42,42 & 30,23 & 45,05 & 6 & 6 \\
\hline Baleares & 2,82 & 2,53 & 2,20 & 2,39 & 2,15 & 2,29 & 8,66 & 3,50 & 3,96 & 2,68 & 8 & 8 \\
\hline Canarias & 4,10 & 8,17 & 5,36 & 10,85 & 3,93 & 6,70 & 3,14 & 9,80 & 4,13 & 8,88 & 7 & 7 \\
\hline
\end{tabular}

A) TOTAL

Revista de Evaluación de Programas y Políticas Públicas | Núm. 4 (2015), pp.59-85 


\section{Cuadro 5}

Índices de atractivo expost (cont.)

\begin{tabular}{|c|c|c|c|c|c|c|c|c|c|c|c|c|}
\hline \multirow{2}{*}{ Fachadas } & \multicolumn{2}{|c|}{ DI } & \multicolumn{2}{|c|}{ D2 } & \multicolumn{2}{|c|}{ D3 } & \multicolumn{2}{|c|}{ D4 } & \multicolumn{2}{|c|}{ Media } & \multicolumn{2}{|c|}{ Prelación } \\
\hline & Valor & Peso & Valor & Peso & Valor & Peso & Valor & Peso & Valor & Peso & Valor & Peso \\
\hline \multicolumn{13}{|c|}{ Contenedores } \\
\hline Galicia & 19,68 & 26,30 & 16,50 & 17,16 & $\mathrm{I}, 5 \mathrm{pt}$ & 23,56 & 19,42 & 33,50 & $18,7 \mid$ & 25,13 & 5 & 5 \\
\hline Norte & 71,60 & 75,03 & 70,76 & 58,06 & 68,38 & 73,35 & 119,09 & 90,84 & 82,46 & 74,32 & 4 & 3 \\
\hline Cataluña & 94,07 & 78,97 & 114,96 & 76,15 & 97,35 & 78,16 & 58,56 & 62,24 & 91,23 & 73,88 & 3 & 4 \\
\hline Levante & 102,05 & 132,03 & 111,93 & $|47,9|$ & 111,29 & 135,28 & 60,65 & 124,69 & 96,48 & 134,98 & 2 & I \\
\hline Surmediterránea & 174,97 & 97,44 & 117,29 & 56,59 & $|56,8|$ & 87,37 & $27 I, 06$ & 149,76 & 180,03 & 97,79 & I & 2 \\
\hline Suratlántica & 17,78 & 20,58 & 16,10 & 15,47 & 18,42 & 20,37 & 10,74 & 20,29 & 15,76 & 19,18 & 6 & 6 \\
\hline Baleares & 0,00 & 0,00 & 0,00 & 0,00 & 0,00 & 0,00 & 0,00 & 0,00 & 0,00 & 0,00 & 8 & 8 \\
\hline Canarias & $|, 7|$ & 1,38 & $\mathrm{I}, 07$ & 0,83 & $|, 7|$ & 1,30 & 1,60 & 1,96 & 1,52 & $\mathrm{I}, 37$ & 7 & 7 \\
\hline \multicolumn{13}{|c|}{ Graneles líquidos } \\
\hline Galicia & 16,65 & 24,60 & 28,24 & 35,32 & 15,49 & 22,43 & 17,53 & 25,53 & 19,48 & 26,97 & 6 & 7 \\
\hline Norte & 53,43 & 49,58 & 82,38 & 95,02 & 56,63 & 49,58 & 42,15 & 62,31 & 58,65 & 64,12 & 4 & 4 \\
\hline Cataluña & 180,47 & 193,48 & 147,62 & $|44,0|$ & 180,95 & 202,92 & 157,50 & 108,92 & 166,63 & 162,33 & 1 & 2 \\
\hline Levante & 63,09 & 70,47 & 73,11 & 71,17 & 65,84 & 73,95 & 43,14 & 45,71 & 61,30 & 65,32 & 3 & 3 \\
\hline Surmediterránea & 155,24 & 153,07 & 143,60 & 137,63 & 151,75 & 145,75 & 194,85 & 220,15 & 161,36 & 164,15 & 2 & 1 \\
\hline Suratlántica & 19,89 & 26,11 & 38,34 & 41,10 & 18,73 & 24,70 & 20,41 & 24,49 & 24,34 & 29,10 & 5 & 6 \\
\hline Baleares & 0,00 & 0,00 & 0,00 & 0,00 & 0,00 & 0,00 & 0,00 & 0,00 & 0,00 & 0,00 & 8 & 8 \\
\hline Canarias & 17,73 & 29,79 & 17,12 & 23,71 & 14,55 & 24,38 & 26,62 & 40,20 & 19,00 & 29,52 & 7 & 5 \\
\hline \multicolumn{13}{|c|}{ Graneles sólidos } \\
\hline Galicia & 287,65 & 261,84 & 190,87 & $|46,6|$ & 280,73 & $24 I, 65$ & 292,36 & 354,29 & 262,90 & 251,10 & 1 & $\mathrm{I}$ \\
\hline Norte & 87,92 & 119,60 & 118,30 & 148,07 & 88,83 & 125,53 & 96,27 & 93,55 & 97,83 & 121,69 & 2 & 2 \\
\hline Cataluña & 39,02 & 41,38 & 44,14 & 43,49 & 43,84 & 42,12 & 17,79 & 29,63 & 36,20 & 39,15 & 4 & 6 \\
\hline Levante & 16,31 & 44,16 & 33,20 & 58,25 & 18,15 & 47,46 & 13,10 & 32,02 & 20,19 & 45,47 & 6 & 5 \\
\hline Surmediterránea & 24,13 & 54,34 & 25,43 & 61,01 & 27,71 & 56,80 & 11,52 & 43,09 & 22,20 & 53,81 & 5 & 3 \\
\hline Suratlántica & 49,43 & 43,09 & 71,93 & 62,18 & 50,02 & 42,56 & 46,18 & 45,24 & 54,39 & 48,27 & 3 & 4 \\
\hline Baleares & 0,02 & 0,17 & 0,07 & 0,52 & 0,02 & 0,16 & 0,03 & 0,35 & 0,04 & 0,30 & 7 & 7 \\
\hline Canarias & 0,00 & 0,00 & 0,00 & 0,00 & 0,00 & 0,00 & 0,00 & 0,00 & 0,00 & 0,00 & 8 & 8 \\
\hline \multicolumn{13}{|c|}{ Mercancía general } \\
\hline Galicia & 57,89 & 77,19 & 64,58 & 76,37 & 61,25 & 72,77 & 45,41 & 112,86 & 57,28 & 84,80 & 5 & 3 \\
\hline Norte & 157,85 & 151,28 & 142,64 & 154,30 & $|4|, 7 \mid$ & 148,66 & 226,06 & 154,99 & 167,07 & $|52,3|$ & 1 & 1 \\
\hline Cataluña & 90,26 & 81,55 & 112,36 & 89,65 & $102,7 \mid$ & 85,20 & 50,65 & 75,18 & 88,99 & 82,90 & 3 & 4 \\
\hline Levante & 86,76 & 93,36 & 82,52 & 90,02 & 78,24 & 94,01 & 107,29 & 83,15 & 88,70 & 90,13 & 4 & 2 \\
\hline Surmediterránea & 110,76 & 90,56 & 89,23 & 69,18 & 120,77 & 98,64 & 56,71 & 45,53 & 94,36 & 75,98 & 2 & 5 \\
\hline Suratlántica & 20,28 & 20,32 & 18,10 & 24,48 & 22,12 & 21,89 & 11,12 & 11,29 & 17,91 & 19,49 & 6 & 6 \\
\hline Baleares & 4,17 & 5,99 & 3,98 & 3,81 & 4,16 & 5,78 & 6,55 & 10,32 & 4,72 & 6,47 & 7 & 7 \\
\hline Canarias & 0,96 & 2,96 & 1,05 & 3,38 & 1,00 & 3,00 & 0,68 & 2,28 & 0,92 & 2,91 & 8 & 8 \\
\hline \multicolumn{13}{|c|}{ Total } \\
\hline Galicia & 43,52 & 83,03 & 41,09 & 59,84 & 43,91 & 77,64 & 36,88 & 105,20 & 41,35 & 81,43 & 5 & 5 \\
\hline Norte & 112,77 & 112,54 & 105,16 & 114,98 & 102,44 & $|10,6|$ & 176,59 & 130,07 & 124,24 & 117,05 & 2 & 2 \\
\hline Cataluña & 99,50 & 100,03 & 121,10 & 101,24 & 107,14 & 102,60 & 59,24 & 79,66 & 96,74 & 95,88 & 3 & 4 \\
\hline Levante & 97,15 & 117,49 & 103,30 & 134,34 & 99,23 & 121,15 & 86,06 & 104,07 & 96,44 & 119,26 & 4 & 1 \\
\hline Surmediterránea & 151,73 & $109,8 \mid$ & || $2,7 \mid$ & 86,00 & 146,83 & 106,30 & 169,22 & 120,90 & 145,12 & 105,75 & 1 & 3 \\
\hline Suratlántica & 20,62 & 27,83 & 20,33 & 32,98 & 21,72 & 28,24 & 12,12 & 21,92 & 18,70 & 27,74 & 6 & 6 \\
\hline Baleares & 1,83 & 1,82 & 1,57 & 1,04 & 1,73 & 1,60 & 3,33 & 4,82 & 2,11 & 2,32 & 7 & 8 \\
\hline Canarias & 2,07 & 5,52 & 1,96 & 5,55 & 2,03 & 4,86 & 1,83 & 6,97 & 1,97 & 5,73 & 8 & 7 \\
\hline
\end{tabular}

B) EXPORTACIONES

Revista de Evaluación de Programas y Políticas Públicas | Núm. 4 (2015), pp.59-85 


\section{Cuadro 5}

Índices de atractivo expost (cont.)

\begin{tabular}{|c|c|c|c|c|c|c|c|c|c|c|c|c|}
\hline \multirow{2}{*}{ Fachadas } & \multicolumn{2}{|c|}{ DI } & \multicolumn{2}{|c|}{ D2 } & \multicolumn{2}{|c|}{ D3 } & \multicolumn{2}{|c|}{ D4 } & \multicolumn{2}{|c|}{ Media } & \multicolumn{2}{|c|}{ Prelación } \\
\hline & Valor & Peso & Valor & Peso & Valor & Peso & Valor & Peso & Valor & Peso & Valor & Peso \\
\hline \multicolumn{13}{|c|}{ Contenedores } \\
\hline Galicia & 31,78 & 21,32 & 20,60 & $|9,5|$ & $24,4 I$ & 16,92 & 58,56 & 41,68 & 33,84 & 24,86 & 5 & 6 \\
\hline Norte & 84,31 & 72,39 & 54,45 & 59,23 & 70,93 & 62,14 & 151,13 & || $6,8 \mid$ & 90,21 & 77,64 & 3 & 3 \\
\hline Cataluña & 104,49 & 82,32 & 125,05 & 92,80 & 100,15 & 76,78 & 107,92 & $|I|, 5 \mid$ & 109,40 & 90,85 & 2 & 2 \\
\hline Levante & 137,86 & 163,42 & 121,85 & 164,99 & 153,72 & 174,29 & 100,08 & 115,85 & 128,38 & 154,64 & 1 & I \\
\hline Surmediterránea & 67,52 & 71,12 & 40,79 & 48,62 & 64,06 & 65,07 & 63,09 & 80,60 & 58,86 & 66,35 & 4 & 4 \\
\hline Suratlántica & 20,18 & 22,16 & 10,42 & 14,29 & 13,77 & 15,93 & 56,90 & 51,30 & 25,32 & 25,92 & 6 & 5 \\
\hline Baleares & 0,00 & 0,00 & 0,00 & 0,00 & 0,00 & 0,00 & 0,00 & 0,00 & 0,00 & 0,00 & 8 & 8 \\
\hline Canarias & 4,65 & 5,55 & 4,95 & 6,83 & 4,50 & 5,02 & 4,01 & 5,40 & 4,53 & 5,70 & 7 & 7 \\
\hline \multicolumn{13}{|c|}{ Graneles líquidos } \\
\hline Galicia & 43,77 & 46,20 & 40,18 & 43,56 & 42,19 & 42,54 & 72,04 & 87,70 & 49,54 & 55,00 & 5 & 5 \\
\hline Norte & 86,75 & 83,09 & 84,56 & 86,02 & 86,12 & 79,10 & 131,23 & 140,53 & 97,16 & 97,18 & 4 & 3 \\
\hline Cataluña & 107,87 & 95,20 & 129,10 & 118,15 & 113,18 & 98,42 & 95,43 & 68,99 & 111,39 & 95,19 & 3 & 4 \\
\hline Levante & 137,55 & 142,46 & 142,79 & 151,10 & $|38,3|$ & 153,16 & 108,45 & 98,59 & 131,78 & 136,33 & 2 & 2 \\
\hline Surmediterránea & 151,19 & 171,54 & 100,45 & 103,77 & 142,34 & 155,56 & 159,28 & 198,08 & 138,32 & 157,24 & 1 & 1 \\
\hline Suratlántica & 35,95 & 41,62 & 48,95 & 55,32 & 37,47 & 42,81 & 26,60 & 32,31 & 37,24 & 43,02 & 6 & 6 \\
\hline Baleares & 8,18 & 6,85 & 6,58 & 5,39 & 8,23 & 6,46 & 7,61 & 8,83 & 7,65 & 6,88 & 8 & 8 \\
\hline Canarias & 21,60 & $21,4 \mid$ & 22,15 & 23,13 & 18,50 & 16,95 & 23,86 & 28,59 & 21,53 & 22,52 & 7 & 7 \\
\hline \multicolumn{13}{|c|}{ Graneles sólidos } \\
\hline Galicia & 113,56 & 119,70 & 80,99 & 90,85 & 108,94 & 115,89 & 108,15 & 108,16 & 102,91 & 108,65 & 2 & 2 \\
\hline Norte & 195,49 & $|49,0|$ & 181,93 & 158,60 & 197,86 & 149,68 & 190,50 & $|5|, 3 \mid$ & 191,45 & 152,15 & 1 & 1 \\
\hline Cataluña & 36,58 & 45,17 & 50,95 & 60,44 & 34,20 & 43,43 & 51,03 & 52,14 & 43,19 & 50,30 & 4 & 6 \\
\hline Levante & 35,64 & 100,44 & 50,26 & 98,04 & 35,61 & 103,04 & 31,81 & 92,43 & 38,33 & 98,49 & 5 & 3 \\
\hline Surmediterránea & 30,39 & 58,23 & 45,65 & 51,12 & 30,93 & 60,74 & 36,48 & 62,19 & 35,86 & 58,07 & 6 & 5 \\
\hline Suratlántica & 63,13 & 60,89 & 75,52 & 59,16 & 62,85 & 59,83 & 62,80 & 68,33 & 66,07 & 62,05 & 3 & 4 \\
\hline Baleares & 0,32 & 0,58 & 0,75 & 1,20 & 0,32 & 0,58 & 0,31 & 0,69 & 0,42 & 0,77 & 8 & 8 \\
\hline Canarias & 0,94 & 1,88 & 1,72 & 4,79 & 0,80 & 1,22 & 1,27 & 4,12 & 1,18 & 3,00 & 7 & 7 \\
\hline \multicolumn{13}{|c|}{ Mercancía general } \\
\hline Galicia & 23,21 & 34,15 & 27,46 & 44,86 & 19,73 & 33,84 & 44,65 & 30,09 & 28,76 & 35,73 & 6 & 5 \\
\hline Norte & 133,57 & 134,40 & 137,49 & 144,04 & 129,57 & 135,63 & 147,70 & 134,27 & 137,08 & 137,09 & 2 & 1 \\
\hline Cataluña & 68,02 & 121,40 & 76,34 & 92,50 & 67,40 & 111,33 & 65,04 & 165,88 & 69,20 & 122,78 & 4 & 2 \\
\hline Levante & 67,45 & 99,58 & 71,69 & 111,40 & 55,56 & 103,33 & 107,99 & 80,99 & 75,67 & 98,82 & 3 & 4 \\
\hline Surmediterránea & 177,94 & 109,40 & 159,70 & 77,45 & 196,55 & 110,04 & 119,46 & 101,17 & $163,4 \mid$ & 99,51 & 1 & 3 \\
\hline Suratlántica & 38,34 & 35,45 & 35,56 & 35,62 & 35,24 & 38,30 & 30,81 & 24,28 & 34,99 & 33,41 & 5 & 6 \\
\hline Baleares & 5,55 & 2,02 & 4,27 & 1,32 & 3,20 & 1,08 & 18,67 & 2,29 & 7,92 & 1,68 & 7 & 8 \\
\hline Canarias & 1,20 & 2,64 & 2,07 & 2,83 & 1,33 & 2,55 & 0,68 & 2,10 & 1,32 & 2,53 & 8 & 7 \\
\hline \multicolumn{13}{|c|}{ Total } \\
\hline Galicia & 41,35 & 76,07 & 34,84 & 64,77 & 37,15 & 73,38 & 58,83 & 75,61 & 43,04 & 72,46 & 5 & 5 \\
\hline Norte & 128,50 & 125,99 & 105,93 & 128,75 & 121,64 & 124,09 & 161,92 & 144,33 & 129,50 & 130,79 & 1 & 1 \\
\hline Cataluña & 97,02 & 84,17 & 122,25 & 96,63 & 96,86 & 81,87 & 88,48 & 98,13 & 101,15 & 90,20 & 4 & 4 \\
\hline Levante & 116,36 & 133,72 & 121,33 & $|38,3|$ & 122,15 & 141,58 & 109,92 & 100,40 & 117,44 & 128,50 & 3 & 2 \\
\hline Surmediterránea & 132,72 & 109,95 & 95,62 & 78,89 & 136,73 & 105,66 & 106,07 & 114,00 & 117,79 & 102,13 & 2 & 3 \\
\hline Suratlántica & 36,92 & 49,38 & 32,72 & 53,59 & 33,05 & 49,13 & 44,71 & 48,02 & 36,85 & 50,03 & 6 & 6 \\
\hline Baleares & 3,48 & 2,75 & 2,59 & 2,77 & 2,45 & 2,50 & 11,22 & 3,11 & 4,93 & 2,78 & 8 & 8 \\
\hline Canarias & 5,46 & 8,97 & 7,53 & 12,32 & 5,29 & 7,26 & 3,75 & 10,56 & 5,51 & 9,78 & 7 & 7 \\
\hline
\end{tabular}

C) IMPORTACIONES

Revista de Evaluación de Programas y Políticas Públicas | Núm. 4 (2015), pp.59-85 


\section{Cuadro 6}

Coeficientes de correlación valor-peso.

\begin{tabular}{|l|c|c|c|c|}
\hline & D & D2 & D3 & D4 \\
\hline \multicolumn{5}{|c|}{ Total } \\
\hline Contenedores & 0,95 & 0,92 & 0,96 & 0,92 \\
\hline Graneles Líquidos & 0,99 & 0,99 & 0,98 & 0,95 \\
\hline Graneles Sólidos & 0,91 & 0,94 & 0,90 & 0,91 \\
\hline Mercancía General & 0,91 & 0,90 & 0,89 & 0,83 \\
\hline Total & 0,93 & 0,92 & 0,92 & 0,96 \\
\hline \multicolumn{5}{|c|}{ Exportaciones } \\
\hline Contenedores & 0,86 & 0,84 & 0,88 & 0,85 \\
\hline Graneles Líquidos & 1,00 & 0,99 & 0,99 & 0,95 \\
\hline Graneles Sólidos & 0,98 & 0,92 & 0,97 & 0,99 \\
\hline Mercancía General & 0,98 & 0,97 & 0,97 & 0,86 \\
\hline Total & 0,91 & 0,93 & 0,92 & 0,85 \\
\hline \multicolumn{7}{|c|}{ Importaciones } & & 0,94 \\
\hline Contenedores & 0,97 & 0,93 & 0,98 & 0,96 \\
\hline Graneles Líquidos & 0,99 & 0,99 & 0,99 & 0,90 \\
\hline Graneles Sólidos & 0,88 & 0,93 & 0,87 & 0,76 \\
\hline Mercancía General & 0,83 & 0,80 & 0,80 & 0,98 \\
\hline Total & 0,94 & 0,92 & 0,93 & \\
\hline
\end{tabular}

\section{Conclusiones}

En este trabajo se ha abordado el análisis de la competencia y competitividad de las fachadas marítimas españolas, para lo cual se han examinado los datos (en valor y peso) del tráfico total, exportaciones e importaciones, desglosados según el tipo de mercancía transportada. Tras una revisión de la literatura sobre este tema, con especial referencia al caso español, se han calculado tres índices de atractivo portuario alternativos, todos ellos basados en los datos de tráfico recogidos por cada una de las fachadas en el año 2007.

De acuerdo con los resultados obtenidos, parece que las fachadas marítimas con mayor atractivo son la Surmediterránea, si hablamos en términos de valor, y la Norte, si lo hacemos en términos de peso. Los resultados se mantienen, grosso modo, cuando diferenciamos entre exportaciones e importaciones. En lo relativo a la forma de presentación de las mercancías comerciadas, e independientemente de que consideremos su valor o peso, la fachada Norte ocupa el primer lugar en graneles sólidos y mercancía general, mientras que la Surmediterránea ocupa tal posición en lo que se refiere a graneles líquidos, al tiempo que Levante sobresale como la fachada más atractiva en el tráfico de contenedores.

Para concluir, podemos preguntamos qué recomendaciones sobre políticas públicas pueden extraerse de los resultados de este trabajo. Pues bien, en este sentido, el tan conocido dilema «equidad-eficiencia» entra, como casi siempre, en juego. Más aún cuando, como hemos visto en el trabajo, las diferencias entre unidades (fachadas en este caso) 
son muy significativas. Si, en las condiciones económicas actuales, consideramos que prevalece la búsqueda de la eficiencia, de este trabajo puede colegirse la conveniencia de incrementar las inversiones públicas en los puertos pertenecientes a las fachadas Norte y Surmediterránea, especialmente en los factores más relacionados con el medio de transporte en el que se muestran más competitivas. Por otro lado, y aunque quizás no sea el momento más adecuado por la situación económica que vive el país, no podemos olvidad el factor equidad en las políticas públicas, con lo que ayudas futuras para, por ejemplo, las fachadas insulares (lastradas en términos competitivos) serían muy bien recibidas.

Sea cual sea el caso, de forma genérica las inversiones deberían ir destinadas a una mejora en las infraestructuras (desarrollando, por ejemplo, la intermodalidad para integrar mejor los distintos medios de transporte a la cadena logística del puerto) y a potenciar la innovación como fuente de creación de valor, de mejora de eficiencia y de incremento en la calidad/diferenciación de los servicios portuarios. En definitiva, mejorar la infraestructura y promover la innovación como instrumentos para incrementar la productividad portuaria que permitan la puesta en valor de los activos de cada puerto en beneficio de su entorno, tanto empresarial como social.

Por último, una breve mención a la conveniencia de políticas públicas que exploten las potenciales sinergias entre los puertos de cada fachada. En efecto, resulta obvio apuntar que otra forma de incrementar la eficiencia de cada fachada sería la implementación de políticas que favoreciesen la colaboración entre los puertos integrantes de la misma. La conjunción de colaboración y competencia sería, muy probablemente, la mejor forma de ganar competitividad.

\section{Referencias Bibliográficas}

Baños-Pino, J., Coto Millán, P. \& Rodríguez-Álvarez, A. (1999). Allocative efficiency and over-capitalization: an application. InternationalJournal of Transport Economics, 26(2), 181-199.

Becchetti, 1., Bedoya, D. \& Paganetto, 1. (2003). ICT Investment, Productivity and Efficiency: Evidence at Firm Level Using a Stochastic Frontier Approach. Journal of Productivity Analysis, 20, 143-167.

Beham, S. (1988). Economic Impact of Dublin Port on its Hinterland. Dublin: Dublin Port.

Budd, L. \& Hirmis, A. (2004). Conceptual framework for regional competitiveness. Regional Studies, 38(9), 1001-1014.

Castillo-Manzano, J.I., Coto-Millán, P., Pesquera, M.A. \& López-Vapuesta, L. (2004). Comparative Analysis of Port Economic Impact Studies in the Spanish Port System (1992-2000). En Essays on Microeconomics and Industrial Organization, Second Edition (Chapter 19, pp. 297-316). Alemania: Physica- Verlag, Springer.

Castillo-Manzano, J.I., Castro-Nuño, M., González-Laxe, F., López-Vapuesta, L. \& Arévalo-Quijada, T. (2009). Low-cost port competitiveness index: Implementation in the Spanish port system. Marine Policy, 33(4), 591-598. 
Cerbán, M. (2009). Competitividad Económica de los Puertos. En Piniella, F. (ed.), Gestión Portuaria y Logística (pp. 73-89), Sevilla: Editorial UIA.

Chang, S. (1978). Production function and capacity utilization of the port of Mobile. Maritime Policy and Management, 5, 297-305.

Comisión Europea (1999). Sexto informe periódico sobre la situación económica y social de las regiones de la UE. EC.

Coto-Millán, P. (2010). Mecanismos de regulación en infraestructuras: una aplicación a los puertos españoles. Mimeo.

Coto Millán, P., Baños-Pino, J. \& Rodríguez-Álvarez, A. (2000). Economic efficiency in Spanish ports: Some empirical evidence. Maritime Policy and Management, 2(2), 169-174.

Coto-Millán, P., Gallego, J.L. \& Villaverde, J. (2001). Crecimiento y Desarrollo Portuario. Aplicación al Puerto de Santander. Santander: Autoridad Portuaria de Santander

Coto-Millán, P., Villaverde, J. \& Mateo, I. (2008). Impacto Económico del Puerto de Santander en la Ciudad, en Cantabria y en otras regiones españolas. Santander: Autoridad Portuaria de Santander.

Crozet, M. (2004). Do migrants follow market potentials? An estimation of a new economic geography model. Journal of Economic Geography, 4, 439-458.

Cullinane, K., Song, D.W. \& Gray, R. (2002). A stochastic frontier model of the efficiency of mayor container terminals in Asia: Assessing the influence of administrative and ownership structures. Transportation Research, 36(A), 743-762.

Cullinane, K., Wang, T.F., Song, D.W. \& Ji, P. (2006). The technical efficiency of container ports: Comparing data development analysis and stochastic frontier analysis. Transportation Research, 4O(A), 354-374.

De Rus, G., Román, C. \& Trujillo, L. (1994). Estimación de la Actividad Económica y Estructura de Costes del Puerto de La Luz y de las Palmas. Las Palmas: Civitas, Autoridad Portuaria de Las Palmas.

Díaz-Hernández, J.J., Martínez-Budría, E. \& Jara-Díaz, S. (2008). Parametric estimation of inefficiency in cargo handling in Spanish ports. Journal of Productivity Analysis, $30(3), 223-232$.

European Commission (2004). A new partnership for cohesion: convergence, competitiveness and cooperation. EC.

Fleming, D. \& Baird, A. (1999). Some reflections on port competition in the United States and Western in Europe. Maritime Policy Management, 26(4), 383-394.

Fraga, J. \& Seijas, J.A. (1992). El Puerto de Ferrol y su influencia en la economía de la comarca. Ferrol: Junta del Puerto y Ría de Ferrol.

García, L. (2005). Competencia interportuaria: delimitación y análisis del área de influencia de los puertos españoles (Tesis doctoral). Universidad de Oviedo. 
García, L., Sánchez, R. \& Vallejo, I. (2007). La competencia interportuaria: Análisis del caso español. En González Laxe, F. y Sánchez, R.J. (eds.), Lecciones de Economía Marítima, Capítulo 6, pp. 165-192. Editorial Netbiblo.

González, M.M. \& Trujillo, L. (2009). Efficiency measurement in the port industry: A survey of the empirical evidence. Journal of Transport Economics and Policy, 43(2), 157-192.

Haezendonck, E. \& Notteboom, T. (2002). The Competitive advantage of seaports. En Winkelmans, W. (coord.). Port Competitiveness . An Economic and legal analysis of the factors determining the competitiveness of seaports. Antwerp: Editorial De Boeck Ltd., Institute of Transport and Maritime Management of Antwerp.

Hidalgo, S., Nuñez-Sánchez, R. \& Coto-Millán, P. (2011). Incertidumbre en la demanda, exceso de capacidad y eficiencia asignativa: Una aplicación a las autoridades portuarias españolas en el periodo 1986-2007 (Documento de Trabajo). Universidad Cantabria.

Huybrechts, M. \& Meersman, CH. (2002). Port Competitiveness. En Winkelmans, W. (coord). Port Competitiveness. An Economic and legal analysis of the factors determining the competitiveness of seaports. Antwerp: Editorial De Boeck Ltd., Institute of Transport and Maritime Management of Antwerp.

Jara-Díaz, S., Cortes, C., Vargas, A. \& Martínez-Budría, E. (1997). Marginal Costs and Scale Economies in Spanish ports. 25th European Transport Forum, Proceedings Seminal L, PTRC, Londres, pp. 137-147.

Jara-Díaz, S., Martínez-Budría, E., Cortes, C. \& Basso, l. (2002). A multioutput cost function for the services of Spanish ports infrastructure. Transportation, 29(4), 415-437.

Kim, M. \& Sachis, A. (1986). The structure of production, technical change and productivity in a port. International Journal of Industrial Economics, 35(2), 209223.

Kitson, M., Martin, R. \& Tyler, P. (2004). Regional competitiveness: An elusive yet key concept. Regional Studies, 38(9), 991-999.

Martínez-Budría, E. (1996). Un estudio econométrico de los costes del sistema portuario español. Revista Asturiana de Economía, 16, 135-149.

Martínez-Budría, E., Díaz-Hernández, J. \& Jara-Díaz, S. (1998). Análisis económico de las sociedades estatales de estiba y desestiba (Documento de trabajo 97/98.1). Universidad de La Laguna.

Mateo, I. (2010). Impacto económico por tipo de mercancía y huella ecológica en Puertos (Tesis doctoral). Universidad de Cantabria.

Mateo, I., Coto-Millán, P. \& Villaverde, J. (2012). Economic impact of a port on the hinterland: Application to Santander's port. International Journal of Shipping and Transport Logistics, 4(3), 235-249. 
Neumann, J.V. (1944). Theory of Games and Economic Behavior. Princeton: The Arts Publisher, Princeton University Press.

Noorda, R. (1993). Co-opetition. Electronic Business Buyer, Diciembre, pp. 8-12.

Núñez-Sánchez, R. \& Coto-Millán, P. (2010). The impact of public reforms on the productivity of the Spanish ports: A parametric distance function approach (Documento de trabajo ${ }^{0}$ 513/2010). Fundación de las Cajas de Ahorros.

Nuñez-Sánchez, R., Jara-Díaz, S. \& Coto-Millán, P. (2011). Public regulation and passengers importance in port infrastructure costs. Transportation Research-A: Policy and Practice, 45(7), 653-666.

Porter, M. (1990). The Competitive Advantage of Nations. Free Press.

Rekres, R.A., Connell, D. \& Ross, D.I. (1990). The development of a production function for a container terminal in the port of Melbourne. Papers of the Australian Transport Research Forum, 15, 205-218.

Tema (1994a). Elaboración de una Metodología para la evaluación de Impacto de la Actividad Portuaria sobre la Economía.

Tema (1994b). Evaluación de los Impactos de la Actividad de los Puertos de Galicia sobre la Economía de la Región.

Tema (1995). Evaluación de los Impactos de la Actividad de los Puertos de Galicia sobre la Economía Nacional.

Tongzon, J.L. (1993). The Port of Melbourne Authority's pricing policy: its efficiency and distribution implications. Maritime Policy and Management, 2O(3), 197-203.

Tovar, B. (2002). Análisis multiproductivo de los costes de manipulación de mercancías en terminales portuarias. El Puerto de La Luz y de Las Palmas (Tesis Doctoral). Universidad de Las Palmas de Gran Canaria.

Turok, I (2004). Cities, regions, and competitiveness. Regional Studies, 38(9), 10691083.

US Maritime Administration (1979). Port Economic Impact KIT. Washington: Office of Port and Intermodal Development.

US Maritime Administration (1982). The Regional Port Impact Model Handbook. Washington: Office of Port and Intermodal Development.

US Maritime Administration (1995). Economic Impact of the Port Industry on the New York-New Jersey Metropolitan Region. Washington: Economic Impacts Division Office of Economic and Policy Analysis Port Authority of New York \& New Jersey, The Port Department.

Vaello, J. (1998). Competencia entre puertos españoles y europeos. Foro de Puertos del Estado. Ministerio de Fomento. 
Van der Voorde, E. \& Winkelmans, W. (2002). A general introduction to port competition and management. En Winkelmans, W. (coord.), Port Competitiveness . An Economic and legal analysis of the factors determining the competitiveness of seaports. Antwerp: Editorial De Boeck Ltd., Institute of Transport and Maritime Management of Antwerp.

Villaverde, J. \& Coto-Millán, P. (1995). El impacto económico del Puerto de Santander en la economía cántabra. Santander: Autoridad Portuaria de Santander.

Villaverde, J. \& Coto-Millán, P. (1996). Impacto económico portuario: Metodologías para su análisis y aplicación al Puerto de Santander. Santander: Autoridad Portuaria de Santander.

Villaverde, J. \& Coto-Millán, P. (1998). Port Economic Impact: methodologies and application to the Port of Santander. International Journal of Transport Economics, special Issue, Infrastructure Investment and Development, $X X V$ (2), 159-179.

Villaverde, J. \& Coto-Millán, P. (1998). Guest Editor's Introduction. International Journal of Transport Economics, special Issue, Infrastructure Investment and Development, $X X V(2), 109-112$.

Villaverde, J., Coto-Millán, P., Aza, R., Baños, J. \& Canal, J.F. (2004). Impacto de los puertos de Avilés y Gijón en la economía asturiana. Papeles de Economía Española, Comunidades Autónomas, Principado de Asturias, 2O, 207-219.

Villaverde, J. \& Maza, A. (2012). El hinterland de las fachadas marítimas españolas. Papeles de Economía Española, 131, 180-199.

Waters, R.C. (1977). Port impact studies: Practice and assessment. Transportation Review, 16, 14-18.

Winkelmans, W. (2002). Port Competitiveness. An Economic and legal analysis of the factors determining the competitiveness of seaports. Antwerp : Editorial De Boeck Ltd., Institute of Transport and Maritime Management of Antwerp

Yanbing, Y. \& Zhongzhen, J. (2005). Evaluation of competition ability and market share for container port. Proceedings of the Eastern Asia Society for Transportation Studies, 5, 2483-2493.

Yochum, G.R. \& Agarwal, V.B. (1987). Economic impact of a port on a regional economy. Growth and Change, 18(3), 74-87.

Zubieta, J. (1998). Competencia entre puertos españoles y europeos. Foro de Puertos del Estado. X Congreso Panamericano de Ingeniería de Tránsito y Transporte. Ministerio de Fomento. 